\title{
Predicting Sound Absorption Coefficients of Lightweight Multilayer Curtains using the Equivalent Circuit Method
}

\author{
Reto Pieren, Kurt Heutschi \\ Empa, Swiss Federal Laboratories for Materials Science and Technology, 8600 \\ Duebendorf, Switzerland
}

\begin{abstract}
A theoretical model to predict the sound absorption of lightweight multilayer curtains is presented. The fabric sheets of the curtain are represented by a network of discrete impedances describing the vibration and the airflow through the fabric. It is shown that the equivalent circuit method (EC) with correct modeling of the air cavities by distributed elements and the impedance transfer method (ITM) yield identical impedance relations. Formulas for the oblique incidence and the statistical absorption coefficient for curtains with an acoustically hard backing and without backing, i.e. freely hanging, are deduced. The model was validated by measurements on a set of 24 lightweight, woven fabrics. For the normal incidence absorption coefficient excellent agreement was achieved with mean value and standard deviation of the differences of $0.01 \pm 0.05$. Based on the proposed model the following conclusions for the application of lightweight multilayer curtains can be drawn: (1) Sound-induced vibrations of the fabrics are an acoustically relevant aspect in the design of lightweight curtains. (2) The optimal specific airflow resistances of the individual layers may not be given in a compact analytical form but have to be determined by an optimisation procedure. (3) The sound energy absorbed by curtains placed in the diffuse field of a room is in the same order of magnitude as the absorption by comparable curtains mounted in front of a wall. (4) For given mass and extension, multilayer arrangements perform superiorly to single layer curtains.
\end{abstract}

Email addresses: reto.pieren@empa.ch (Reto Pieren), kurt.heutschi@empa.ch (Kurt Heutschi) 
Keywords:

sound absorption, textiles, curtains, equivalent circuit

PACS: 43.55.Ev, 43.40.Dx

\section{Introduction}

The application of curtains as sound absorbers for room acoustical purposes has several substantial advantages: Curtains are relatively cost-effective, lightweight, flexible, easy to handle and they enable variable room acoustics. Already in 1970 it was reported that the sound absorption of curtains depends on the mounting distance to the wall, the airflow resistance and the surface mass density of the fabric as well as the draping [1]. In 1990 it was phenomenologically shown that the intrinsic parameters of a textile, i.e. its microstructure, has a substantial influence on the sound absorption coefficient [2]. Since then several authors have successfully predicted absorption coefficients of textiles based on geometrical parameters [3][4][5][6].

A thorough theoretical and numerical investigation on the dissipation of acoustical energy in a thin, lightweight, poroelastic sheet is given in [7] where the analytical solution is obtained based on a Helmholtz integral formulation. Parametric studies revealed that thin, lightweight, poroelastic sheets can provide high absorption coefficients - even at low frequencies [7][8].

The influence of the finite mass of lightweight curtains on the absorption coefficient was already discussed in special cases. For a single, freely hanging fabric sheet the absorption coefficients at specific angles of sound incidence are discussed in [9]. A single fabric with a rigidly backed air cavity was discussed in [6] for normal sound incidence. It was concluded that for lightweight curtains the influence of the finite mass on the absorption coefficient should be taken into account.

Structures consisting of multiple infinitely extended, thin sheets separated by extended air cavities have been widely investigated in the context of microperforated plates (MPP) [10] [11]. So called multiple-leaf MPPs can be used in order to broaden the frequency range of absorption or to get rid of the rigid backing[12] [13] [14] [15]. Recently also a combination of MPPs and a permeable membrane has been studied [16]. Different methods to model the acoustical behavior of such multilayer structures have been adopted and compared, e.g. the transfer matrix method (TMM) [17] [18], the impedance transfer method (ITM) [19] [13] [20] and the equivalent circuit method (EC) 
[13] [21] [14]. An alternative approach using a neuronal network was recently applied to nonwoven multilayer absorbers [22].

In this article the equivalent circuit method will be adopted in order to obtain absorption coefficients of multilayer curtain systems. There are two main reasons for this choice: (1) The combined acousto-mechanical system can be well visualized, interpreted and analysed using this method. (2) In the past decades there seems to have been some confusion in the literature about modeling of layered absorber structures by equivalent electrical networks. By improper representation of air cavities in the analogue electrical network, the results may get wrong. Based on comparisons with measurements or other analysis methods, some authors have concluded that the equivalent circuit method is inaccurate in the application of multi-leaf MPPs [13], [21], [12], [23], [20]. Here we show that theses apparent inaccuracies can be avoided by using a correct equivalent network and that the obtained analytical results are identical to other methods such as ITM or TMM.

This article is the continuation of the work published in [6] and [24]. Compared to our previous study [6] the calculation model presented in section 2 has been substantially improved and extended. In this article a more refined model for the airflow through the fabric taking into account the intrayarn airflow and the shape of the pore cross-sections is developed. The formulas for absorbers consisting of multiple fabric sheets are established and recently published formula for statistical absorption coefficients measured in the reverberation chamber are employed. In section 3 a validation of the presented model performed on a large set of 24 different fabrics is accomplished. Absorption coefficients obtained from impedance tube measurements are compared to predicted values. The latter are calculated from geometrical parameters extracted from macroscopic photographs of the fabrics. By using this refined model, the standard deviation of the differences between measured and calculated absorption coefficients could be reduced by a factor of 2 compared to [6]. Section 4 is dedicated to the application of the presented model. Predictions show that the absorption characteristics of a curtain system can be significantly improved by using multiple textile sheets even if the total surface mass density and distance to the wall is kept constant. The article ends with conclusions in section 5 . 


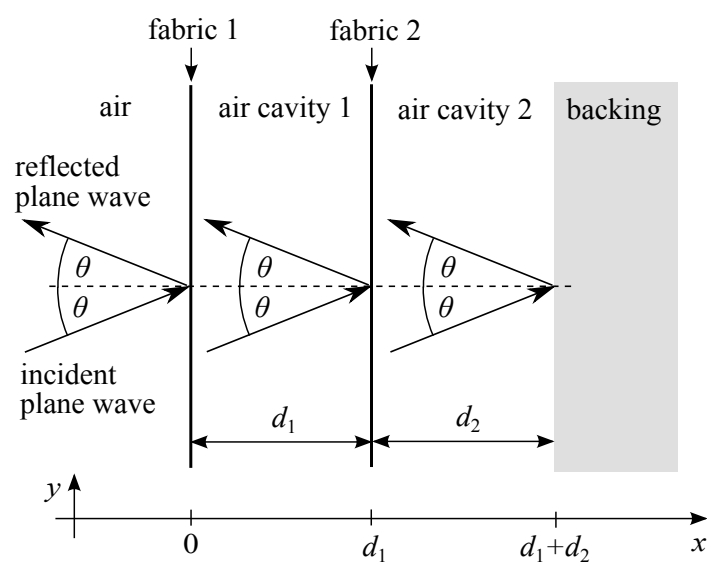

Figure 1: Illustration of a layered absorber consisting of two air layers, two fabric sheets and a backing (grey area).

\section{Model}

In this section a theoretical model to predict sound absorption coefficients of lightweight multilayer curtains is gradually developed using analogue electrical networks. The presented model consists of a comprehensive compilation of already published models and formulas which are combined in order to adequately predict statistical sound absorption coefficients of multilayer curtain systems. The model only considers the predominant acoustical and mechanical effects and merely uses a few easily measurable parameters, which ensures its applicability in the design and optimisation of entire curtain systems.

In a first step the air cavities between the fabric sheets are treated. In a second step the fabric sheets are characterized. Finally the obtained networks are connected and formulas for different absorption coefficients are deduced.

\subsection{Description of the absorbent structure}

The curtain system is assumed to be infinitely extended in two dimensions, $y$ and $z$, and only varying in $x$-direction. This implies that no draping is allowed. Throughout this article generally $0 \%$ fullness is assumed, corresponding to flat curtains. An experimental investigation on the influence of the fullness on statistical sound absorption coefficients can be found in [25]. The curtain consists of multiple, parallel, porous fabric sheets separated by air layers of defined extents. Fig. 1 illustrates a multilayer curtain system 
with two fabric sheets with the corresponding air cavities of extents $d_{1}$ and $d_{2}$. For the backing of the curtain, mainly two cases are of practical relevance: 1) an acoustically hard backing and 2) no backing (i.e. air), which means a freely hanging curtain. It is assumed that a plane wave coming from air impinges upon this structure at an angle $\theta$ to the normal vector of the absorber surface.

\subsection{Analogue electrical networks}

Equivalent electrical networks of acoustical systems can be found by establishing an analogy between the acoustical quantities sound pressure $p$ and sound particle velocity $v$ or volume flow $q$ and the electrical quantities voltage $U$ and current $I$. Widely used is the $p U-v I$ analogy where the potential quantity $p$ is mapped onto $U$ and the flow quantity $v$ or $q$ onto $I$. Similarly mechanical systems can be represented by electrical networks as well. Once the analogy is established, fundamental structures in acoustical and mechnical systems can be identified and described by corresponding electrical network elements.

\subsubsection{Lumped elements}

Short tubes that represent acoustic masses and small cavities that represent acoustic compliances (short and small with respect to the shortest wavelength of interest) can be considered as lumped elements. An important property of lumped elements is the missing of sound propagation due to the element size. Consequently they can be represented by two-poles. The acoustic mass corresponds to an inductance and the compliance to a capacitance as analogue electrical elements.

\subsubsection{Distributed elements representing a long tube}

Distributed elements represent structures that allow for sound propagation. An important and often encountered distributed element is an extended tube-like configuration carrying a plane wave in axial direction. The length of the element is assumed to be $d$ where $d$ is not small compared to the wavelength. A first approach to handle such structures is to subdivide them into smaller sections that fulfill the condition of lumped elements. A long tube can be thought of a pile of cylinders of small height. The air contained in each of these cylinder elements can be accelerated and compressed. These two properties are represented by a serial inductance and a shunt capacitance 


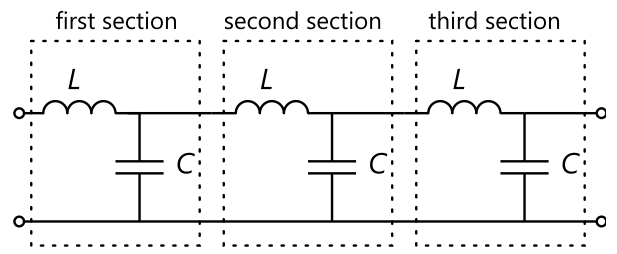

Figure 2: Sequence of three $L C$ elements, each representing a small section of the long tube.

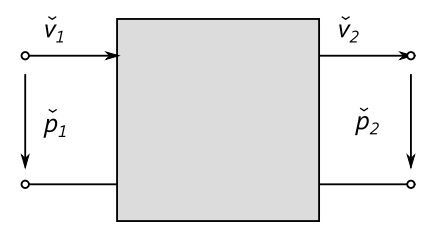

Figure 3: General four-pole that establishes the relations between pressure and velocity at the two ports 1 and 2 .

in the analogue electrical circuit. A sequence of these elements approximates the behavior of the long tube (Fig. 2).

The network in Fig. 2 corresponds to a lossless electrical transmission line. While the behavior of such networks can easily be investigated numerically with help of network analysis tools such as PSPICE, they are not convenient for an analytical treatment. For that purpose it is more helpful to insert distributed elements as four-poles in equivalent networks (Fig. 3).

The four-pole characteristics of a long tube can be derived as follows. A tube allows for two plane waves with pressure amplitudes $A$ and $B$ to travel in opposite directions. The time dependence $\exp (j \omega t)$ is suppressed throughout, where $j$ is the imaginary unit, $\omega$ is the angular frequency, and $t$ is time. The sound field $\check{p}(x)$ and $\check{v}(x)$ as complex amplitude functions can thus be written as

$$
\begin{aligned}
& \check{p}(x)=A e^{-j k x}+B e^{j k x} \\
& \check{v}(x)=\frac{A}{Z} e^{-j k x}-\frac{B}{Z} e^{j k x}
\end{aligned}
$$

where $x$ is the coordinate along the tube axis, $k$ is the wave number and $Z=\rho c$ the acoustical impedance with density $\rho$ and speed of sound $c$. Setting 


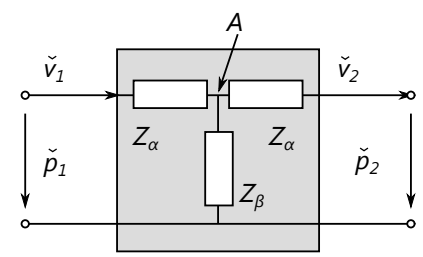

Figure 4: Four-pole as T-type circuit of generalized impedances $Z_{\alpha}$ and $Z_{\beta}$.

$x=0$ at the left port of the four-pole with index 1 and $x=d$ at the right port with index 2, allows to eliminate the pressure amplitudes $A$ and $B$ and express the relations of the four-pole as:

$\check{p}_{2}=\frac{\check{p}_{1}}{2} e^{-j k d}+\frac{\check{p}_{1}}{2} e^{j k d}+\frac{\check{v}_{1} Z}{2} e^{-j k d}-\frac{\check{v}_{1} Z}{2} e^{j k d}=\check{p}_{1} \cosh (j k d)-\check{v}_{1} Z \sinh (j k d)$

$\check{v}_{2}=\frac{1}{Z} \frac{\check{p}_{1}}{2} e^{-j k d}-\frac{1}{Z} \frac{\check{p}_{1}}{2} e^{j k d}+\frac{\check{v}_{1}}{2} e^{-j k d}+\frac{\check{v}_{1}}{2} e^{j k d}=-\check{p}_{1} \frac{1}{Z} \sinh (j k d)+\check{v}_{1} \cosh (j k d)$

It can be shown [26], that the four-pole with the behavior described by Eq. (3) and (4) can be represented by a T-type circuit with generalized impedances (Fig. 4).

The relations between $\check{p}_{1}, \check{p}_{2}, \check{v}_{1}$ and $\check{v}_{2}$ in the T-type circuit from Fig. 4 are found with help of Kirchhoff's circuit laws and impedance relations:

$$
\begin{gathered}
\check{p}_{1}=\check{v}_{1} Z_{\alpha}+\left(\check{v}_{1}-\check{v}_{2}\right) Z_{\beta} \\
\check{p}_{2}=-\check{v}_{2} Z_{\alpha}+\left(\check{v}_{1}-\check{v}_{2}\right) Z_{\beta}
\end{gathered}
$$

To determine the impedances $Z_{\alpha}$ and $Z_{\beta}$, Eq. (5) and (6) are converted into the form of Eq. (3) and (4). From the comparison of the equations follows then

$$
\begin{gathered}
Z_{\alpha}=\frac{Z \sinh (j k d)}{1+\cosh (j k d)}=Z \frac{\cosh (j k d)-1}{\sinh (j k d)} \\
Z_{\beta}=\frac{Z}{\sinh (j k d)}
\end{gathered}
$$


It should be noted that the pressure and the velocities in node $\mathrm{A}$ in the T-type circuit in Fig. 4 are virtual quantities and do not correspond to any field point. As an alternative to the T-type circuit discussed above, a $\Pi$-type circuit can be used instead [26].

\subsection{Application to air cavities in multilayer absorbers}

Multi-layer absorbers consisting of thin sheets separated by large air cavities can be successfully modeled by an equivalent electrical network using the above introduced distributed element for the cavity in form of a T-type circuit. For real-valued wave numbers $k$ which is the case for air $\left(k_{0}=\omega / c_{0}\right)$, $Z_{\alpha}$ and $Z_{\beta}$ from Eq. (7) and (8) can be simplified further

$$
\begin{gathered}
Z_{\alpha}=j Z \frac{1-\cos (k d)}{\sin (k d)} \\
Z_{\beta}=\frac{-j Z}{\sin (k d)}
\end{gathered}
$$

In order to apply these formulas obtained for long tubes to air cavities at oblique sound incidence at an angle $\theta$, the wave number $k$ and the impedance $Z$ have to be adjusted by introducing a factor $\cos \theta$. The formulas for the T-type circuits are then given by

$$
\begin{gathered}
Z_{\alpha}=j \frac{Z_{0}}{\cos \theta} \cdot \frac{1-\cos \left(k_{0} \cos \theta d\right)}{\sin \left(k_{0} \cos \theta d\right)} \\
Z_{\beta}=-j \frac{Z_{0}}{\cos \theta} \cdot \frac{1}{\sin \left(k_{0} \cos \theta d\right)}
\end{gathered}
$$

with the angle of the incident plane wave $\theta$, the wave number in air

$$
k_{0}=\frac{\omega}{c_{0}}
$$

and the impedance of air

$$
Z_{0}=\rho_{0} c_{0}
$$

with the angular frequency $\omega$, speed of sound in air $c_{0}$ and the density of air $\rho_{0}$.

Fig. 5 shows an example with two absorber sheets, such as microperforated plates (MPP) or fabric sheets as illustrated in Fig. 1. $Z_{f, 1}$ and 


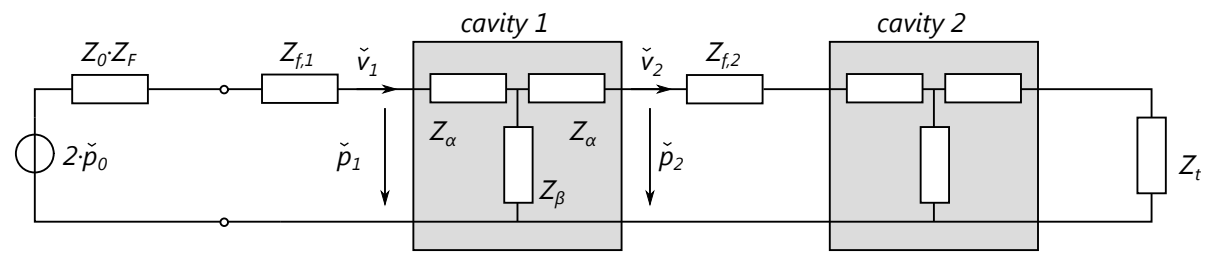

Figure 5: Equivalent electrical circuit for an absorber consisting of two sheets (represented by the two impedances $Z_{f, 1}$ and $Z_{f, 2}$ ) separated by an air cavity (1) and mounted with an air cavity (2) in front of a termination (represented by $Z_{t}$ ).

$Z_{f, 2}$ represent the properties of the sheets, $Z_{t}$ is the termination impedance representing the backing. $\check{p}_{1}$ is the sound pressure on the rear side of sheet $1\left(Z_{f, 1}\right)$ and $\check{p}_{2}$ is the sound pressure on the front side of sheet $2\left(Z_{f, 2}\right)$. For a separating air cavity that is not very small compared to the wavelength follows $\check{p}_{1} \neq \check{p}_{2}$ which makes evident that the air cavity cannot be modeled by a cross impedance to ground as often postulated in literature [10], [27], [3], [13], [14], [23], [28], [29].

\subsection{Comparison of Equivalent Circuit method (EC) and Impedance Transfer Method (ITM)}

In order to compare the equivalent circuit method (EC) and the impedance transfer method (ITM), the surface impedance $Z_{s, 1}$ of a cavity terminated by an impedance $Z_{s, 2} \equiv \check{p}_{2} / \check{v}_{2}$ (Fig. 5) is calculated. Evaluation of the equivalent circuit yields:

$Z_{s, 1} \equiv \frac{\check{p_{1}}}{\check{v}_{1}}=Z_{\alpha}+\frac{Z_{\beta}\left(Z_{\alpha}+Z_{s, 2}\right)}{Z_{s, 2}+Z_{\alpha}+Z_{\beta}}=\frac{Z_{\alpha}\left(Z_{\alpha}+2 Z_{\beta}\right)+\left(Z_{\alpha}+Z_{\beta}\right) Z_{s, 2}}{Z_{s, 2}+\left(Z_{\alpha}+Z_{\beta}\right)}$

With

$$
\left(Z_{\alpha}+Z_{\beta}\right)=-j \frac{Z_{0}}{\cos \theta} \cot \left(k_{0} \cos \theta d\right)
$$

and

$$
Z_{\alpha}\left(Z_{\alpha}+2 Z_{\beta}\right)=\left(\frac{Z_{0}}{\cos \theta}\right)^{2}
$$

follows 


$$
Z_{s, 1}=\frac{Z_{0}}{\cos \theta} \cdot \frac{\frac{Z_{0}}{\cos \theta}-j Z_{s, 2} \cot \left(k_{0} \cos \theta d\right)}{Z_{s, 2}-j \frac{Z_{0}}{\cos \theta} \cot \left(k_{0} \cos \theta\right)}
$$

which is identical to the result obtained by the impedance transfer method (ITM)[19][21]. In contrast to recent published statements [13] [14] [20] [21] the above derivation proves the equivalence of the Equivalent Circuit method (EC) and Impedance Transfer Method (ITM) given that the EC method is correctly applied. Beyond that, the EC method is a more general approach. Whereas the ITM can only be used to calculate impedances, the EC also allows for the calculation of field quantities, transmission coefficients, etc. Alternatively, also the Transfer Matrix Method can be used which yields identical results (see Appendix A).

\subsection{Characterization of fabric sheets}

It is assumed that the fabric sheets are thin compared to the wavelength, e.g. for woven fabrics this typically holds up to $8 \mathrm{kHz}$. In this case no sound propagation inside the sheets has to be modeled and each fabric sheet can be represented by an impedance $Z_{f}$.

\subsubsection{Airflow through the fabric}

It is assumed that the sheets are permeable by air which requires that they have open pores. An excellent short overview of the acoustical effects and their underlying physical phenomena involved in such structures can be found in [30]. The airflow creates a pressure drop across the fabric resulting in an impedance defined as the ratio of the pressure drop and the particle velocity. For static airflow this impedance is given by the specific airflow resistance $R_{s}(\mathrm{~Pa} \mathrm{~s} / \mathrm{m})$ defined by [31]

$$
R_{s} \equiv \frac{\Delta p}{u}
$$

where $\Delta p$ is the pressure difference across the specimen and $u$ is the linear airflow velocity. The specific airflow resistance can be measured directly according to the standard ISO 9053:1991 [31] or determined by an indirect method using an impedance tube [6][32].

The total airflow through the fabric is assumed to consist of two contributions: the interyarn airflow (airflow between yarns) and the intrayarn airflow (airflow through porous yarns), which are both represented by specific airflow resistances $R_{s, r}$ and $R_{s, a}$, respectively. As for the total airflow the 
particle velocities of these airflows are summed up, the total specific airflow resistance, $R_{s}$, corresponds to the parallel circuit of these two elements

$$
R_{s}=R_{s, r} \| R_{s, a}=\frac{R_{s, r} R_{s, a}}{R_{s, r}+R_{s, a}}
$$

In order to predict the static interyarn airflow a capillary model is adopted $[18][5]$

$$
R_{s, r}=\frac{8 \mu \alpha_{\infty} h}{\phi \bar{r}^{2}}
$$

where $\mu, h, \phi, \alpha_{\infty}$ and $\bar{r}$ are the dynamic viscosity of air, the thickness and the interyarn porosity of the fabric, respectively, and the hydraulic radius and the geometrical tortuosity of an open pore, respectively. For circular pores, which can be assumed for knitted fabrics [4] [33] [34], the hydraulic radius equals the geometrical radius. For rectangular pores - which often occur in woven fabrics - of length $a$ and width $b$, the hydraulic radius $\bar{r}$ is given by

$$
\bar{r}=\frac{a b}{a+b}=\frac{a}{\frac{a}{b}+1}
$$

However, if the pore lengths are not small compared to the wavelength, the airflow has to be represented by a transmission line model [35].

The specific airflow resistance of the intrayarn airflow is formulated as

$$
R_{s, a}=\frac{h \sigma}{\beta}
$$

where $\beta$ denotes the portion of the surface area with exposable porous yarns and $\sigma$ is the flow resistivity (in $\mathrm{Pa} \mathrm{s} / \mathrm{m}^{2}$ ) of the porous yarns.

However for non-static airflow, inertial effects of the air moving inside the pores and flow distortions on both sides of the fabric occur. The distortions can be characterized by an equivalent tortuosity [30][5]

$$
\alpha_{\infty, \mathrm{eq}}=1+\frac{2 \epsilon}{h}
$$

with the correction length $\epsilon$ which depends on the geometry and for which different models exist [18]. Taking into account the effect of inertia and flow distortions, the acoustical impedance of a fabric can be written as [30]

$$
Z_{\mathrm{ac}} \equiv \frac{\Delta p}{\check{v}}=R_{s}+j \frac{\omega \rho_{0}}{\phi} h \alpha_{\infty, \mathrm{eq}}\left(1+\frac{\alpha_{\infty, \mathrm{eq}}}{4}\right) .
$$


According to [30] for high flow resistivity thin screens the reactive part of (25) is negligible and $Z_{\mathrm{ac}} \approx R_{s}$. In the following part of this article, this approximation is made and the acoustical impedance of the fabric is merely modeled by the static specific airflow resistance. This means that in the applied model, the airflow only consists of viscous effects - and thermal, inertial and flow distortion effects are neglected.

\subsubsection{Mechanical impedance}

When dealing with lightweight fabrics, also the vibration of the fabric has to be taken into account. According to [6] only for frequencies $f>R_{s} /(\pi m)$ with the surface mass density $m$ (in $\mathrm{kg} / \mathrm{m}^{2}$ ), the influence of the finite mass on the normal incidence absorption coefficient of single fabrics is below 0.1 .

The movement of a thin, elastic plate can be described by the bending wave equation. The equation of motion for the displacement $w$ is given by [36][37][38][39]

$$
D^{\prime} \nabla^{4} w+m \omega^{2} w=\Delta p
$$

with $\nabla^{4}$ being the biharmonic operator and the complex-valued flexural rigidity $D^{\prime}$ of the plate. In case of excitation with a plane wave, the dependency from the $y$-coordinate of the pressure drop $\Delta p$ across the infinitely extended, freely moving plate in (26) equals $\Delta \check{p} e^{j \sin \theta k_{0} y}$. By writing the displacement $w(y)=\check{w}_{0} e^{j \sin \theta k_{0} y}$, the differential equation (26) can be solved. This yields the mechanical impedance - the ratio of pressure difference and velocity - for a freely moving, infinitely extended membrane [18] [26] [38]:

$$
Z_{\text {mech }} \equiv \frac{\Delta p}{\check{v}_{M}}=\frac{\Delta p}{j \omega w}=j \omega m-j \frac{D^{\prime} k_{0}^{4} \sin ^{4} \theta}{\omega} .
$$

However, if doing measurements in the impedance tube it has to be noted that the above mentioned boundary conditions are violated and the mechanical impedance therefore differs from (27). The complex flexural rigidity is given by [36]

$$
D^{\prime}=\frac{E h^{3}(1+j \eta)}{12\left(1-\nu^{2}\right)}
$$

where $E, h, \eta$ and $\nu$ represent the Young's modulus, the thickness, the loss factor and the Poisson ratio of the plate, respectively. 


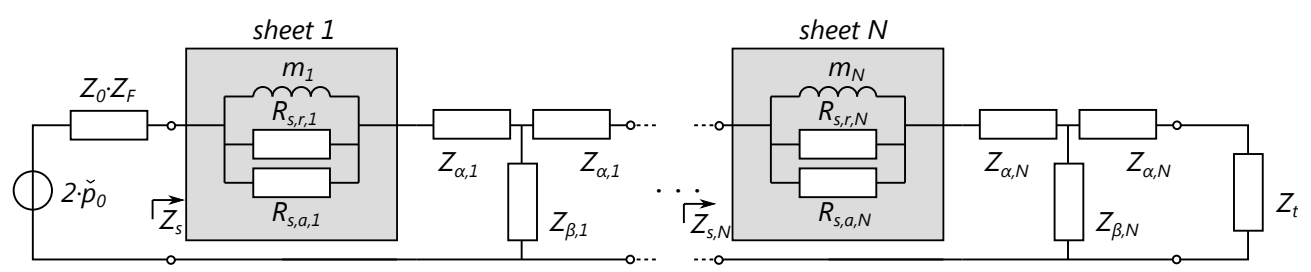

Figure 6: Equivalent electrical circuit of the curtain system with the field in front of the absorber represented as voltage source with source impedance $Z_{0} \cdot Z_{F}$, the fabric sheets as a parallel circuit of specific airflow resistances $R_{s, a, i}$ and $R_{s, r, i}$ and inductor with inductance $m_{i}$, the air layers as T-circuits of lossless transmission lines and the backing represented as a termination impedance $Z_{t}$.

For limp materials, typically the flexural rigidity is very small and often only inertia is taken into account which reduces the mechanical impedance to $j \omega m$ [18][30][6]. In the following sections of this article the flexural rigidity is neglected and the mechanical impedance of the fabric is modeled by $j \omega m$ only.

\subsubsection{Resulting fabric impedance}

The total volume flow through the fabric consists of an acoustical and a mechanical contribution described in sections 2.5.1 and 2.5.2. The resulting particle velocity is thus the sum of the particle velocity inside the fabric and the velocity $v_{M}$ of the moving fabric. In terms of impedances this leads to a parallel circuit of the acoustical and the mechanical impedance

$$
Z_{f}=Z_{\mathrm{ac}} \| Z_{\mathrm{mech}}=\frac{Z_{\mathrm{ac}} Z_{\mathrm{mech}}}{Z_{\mathrm{ac}}+Z_{\mathrm{mech}}}
$$

\subsection{Equivalent electrical circuit (EC) of curtain system}

Fig. 6 shows the equivalent electrical circuit of the system under investigation. The field in front of the absorber is represented by a voltage source, with $\check{p}_{0}$ being the complex pressure amplitude of the incident plane wave, and a source impedance $Z_{0} \cdot Z_{F}$. $Z_{F}$ denotes the field impedance according to [40]. The fabric sheets are represented by a parallel connection of three impedances: two resistances $R_{s, r, i}$ and $R_{s, a, i}$ and an inductance $m_{i}$. The air cavities are represented by T-type circuits of lossless transmission lines according to section 2.3. The backing is represented as a termination 
impedance $Z_{t}$ which for the acoustically hard case is $Z_{t}=\infty$ and for the open case $Z_{t}=Z_{0} / \cos \theta$.

From the equivalent electrical circuit in Fig. 6 the surface impedance which is needed to calculate the absorption coefficient can be extracted. Making use of Eq. (18) yields the recursive expression

$$
Z_{s, i}=Z_{f, i}+\frac{Z_{0}}{\cos \theta} \frac{\frac{Z_{0}}{\cos \theta}-j Z_{s, i+1} \cot \left(k_{0} \cos \theta D_{i}\right)}{Z_{s, i+1}-j \frac{Z_{0}}{\cos \theta} \cot \left(k_{0} \cos \theta D_{i}\right)}
$$

Eq. (30) can now be repeatedly applied in order to obtain the angledependent surface impedance $Z_{s}(\theta)=Z_{s, 1}$ of the multilayer curtain system. The starting condition is given by the termination impedance as $Z_{s, N+1}=$ $Z_{t}$. Note that Eq. (30) obtained from the equivalent electrical circuit is identical to the expression obtained from the impedance transfer method or the transfer matrix method (see Appendix A), respectively.

The fabric impedances $Z_{f, i}$ in Eq. (30) are determined by using Eq. (29), (20) and (27). If the flexural rigidity of the fabric and the reactive part of the acoustical fabric impedance are neglected

$$
Z_{f, i}=R_{s, a, i}\left\|R_{s, r, i}\right\| j \omega m_{i}=\left[\frac{1}{R_{s, a, i}}+\frac{1}{R_{s, r, i}}+\frac{1}{j \omega m_{i}}\right]^{-1}
$$

By using the total specific airflow resistance $R_{s, i}$ the fabric impedance [30][39] [6]

$$
Z_{f, i}=\frac{j \omega m_{i} R_{s, i}}{j \omega m_{i}+R_{s, i}}
$$

which can equivalently be represented by a transfer function-like expression

$$
Z_{f, i}=R_{s, i} \frac{j \frac{\omega}{\omega_{c, i}}}{1+j \frac{\omega}{\omega_{c, i}}}
$$

with the cutoff frequency

$$
f_{c, i}=\frac{\omega_{c, i}}{2 \pi}=\frac{1}{2 \pi} \cdot \frac{R_{s, i}}{m_{i}} .
$$




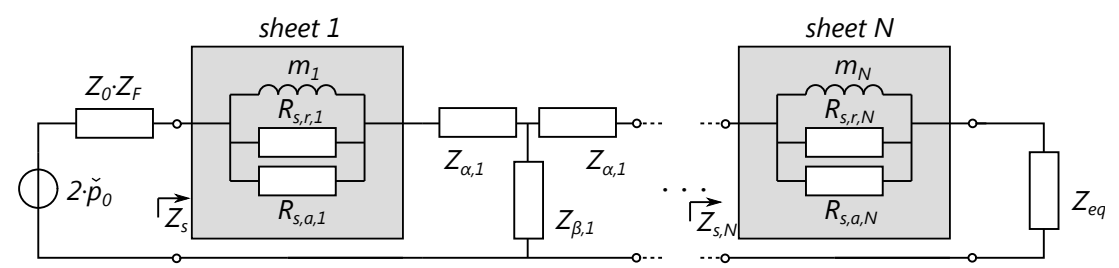

Figure 7: Equivalent electrical circuit of the curtain system with the field in front of the absorber represented as voltage source with source impedance, the fabric sheets as a parallel circuit of specific airflow resistances $R_{s, a, i}$ and $R_{s, r, i}$ and inductor with inductance $m_{i}$, the air layers as T-circuits of lossless transmission lines and the field behind sheet $N$ represented by $Z_{\text {eq }}$.

\subsubsection{Substitution of the backing structure}

For a perfectly rigid backing, the termination impedance $Z_{t}$ becomes infinitely large. In this case the T-type circuit of the last cavity in front of the backing reduces to a series arrangement of $Z_{\alpha, N}$ and $Z_{\beta, N}$. The equivalent impedance $Z_{\text {eq }}$ of this cavity and the rigid termination is given as the sum of the two impedances:

$$
Z_{\text {eq, closed }}=Z_{\alpha, N}+Z_{\beta, N}=-j \frac{Z_{0}}{\cos \theta} \cot \left(k_{0} \cos \theta d_{N}\right)
$$

which is the well-known formula for the surface impedance of a rigidly terminated air cavity. This means that a rigidly backed air cavity can be substituted by an impedance $Z_{\text {eq }}$, which reduces the equivalent electrical circuit as illustrated in Fig. 7.

For the case without backing, i.e. air, the termination impedance is given as $Z_{t}=Z_{0} / \cos \theta$. As no confined air cavity behind sheet $N$ exists, $d_{N}$ can arbitrarily be set to 0 . Consequently the equivalent impedance representing the field behind sheet $N$ yields

$$
Z_{\text {eq,open }}=Z_{0} / \cos \theta \text {. }
$$

\subsection{Absorption coefficient}

For an acoustically hard backing, there is no volume flow into the backing. Under this condition the absorption coefficient, defined as the ratio of the absorbed power, $\Pi_{\mathrm{abs}}$, and the (fictive) incident power, $\Pi_{\text {inc }}$, can be calculated from the surface impedance, $Z_{s}$, of the structure. The oblique incidence 
absorption coefficient of an infinitely extended absorber, i.e. $Z_{F}=1 / \cos \theta$ [40], is given by

$$
\alpha_{\theta} \equiv \frac{\Pi_{\mathrm{abs}}(\theta)}{\Pi_{\mathrm{inc}}(\theta)}=\frac{4 \Re\left\{\tilde{Z}_{s}\right\}}{\left|\tilde{Z}_{s}+1\right|^{2}}
$$

with $\tilde{Z}_{s}=Z_{s}(\theta) /\left(Z_{0} / \cos \theta\right)$. $\Pi_{\text {abs }}$ equals the real power consumed by $Z_{s}$ and $\Pi_{\text {inc }}$ is the real power consumed by $Z_{s}$ under free field conditions $\left(Z_{s}=Z_{0} / \cos \theta\right)$, i.e.

$$
\Pi_{\text {inc }}=\frac{S\left|\check{p}_{0}\right|^{2}}{Z_{0}} \cos \theta
$$

with $S$ being the surface area of the absorber. For the prediction of the statistical absorption coefficient measured in the reverberation chamber, several theoretical and empirical models exist. For Paris'[41][42], Thomasson's[40] and Jeong's[43] formula, the basic equation is given by

$$
\begin{aligned}
\alpha_{s} & =\frac{\int_{0}^{\pi / 2} \Pi_{\mathrm{abs}}(\theta) \sin \theta d \theta}{\int_{0}^{\pi / 2} \Pi_{\mathrm{inc}}(\theta) \sin \theta d \theta} \\
& =2 \int_{0}^{\pi / 2} w(\theta) \frac{4 \Re\left\{Z_{s}^{\prime}(\theta)\right\}}{\left|Z_{s}^{\prime}(\theta)+\bar{Z}_{F}(\theta)\right|^{2}} \sin \theta d \theta,
\end{aligned}
$$

with the normalized surface impedance $Z_{s}^{\prime}=Z_{s} / Z_{0}$, the averaged radiation impedance $\bar{Z}_{F}$ and with $w(\theta)$ being a weighting function. For an infinitely extended absorber, i.e. $Z_{F}=1 / \cos \theta$, and random sound incidence, i.e. $w(\theta)=1$, Eq. (39) becomes the famous Paris' formula [41][42]. However, under real measurement conditions the finite size of the specimen leads to the so called edge-effect. For the statistical absorption coefficient according to Thomasson [40] this effect is considered by using the radiation impedance $\bar{Z}_{F}$ of the specimen. For measurements performed in a reverberation room according to the ISO standard 354 [44] the specimen typically is a rectangle of size $3 \times 4 \mathrm{~m}$. In [45] a method to efficiently calculate the real part of $Z_{F}$ for rectangles is developed. In Appendix B this method is extended for complex values of $Z_{F}$.

Recently, Jeong [43] published frequency dependent angle weighting functions $w(\theta)$ accounting for the non-uniform sound intensity distribution close 
to room surfaces under measurement conditions. Jeong's weighting functions obtained from simulation results can be summarized and approximated by

$$
w(\theta) \cong \begin{cases}1.64-0.3 \cdot \theta-0.47 \cdot \theta^{2}, & f \geq 350 \mathrm{~Hz} \\ 1, & f<350 \mathrm{~Hz}\end{cases}
$$

If the absorber is placed on a room surface, the equivalent sound absorption area is calculated by [44]

$$
A=S \cdot \alpha_{s}
$$

with $S$ being the surface area of the absorber.

If sound transmission into the backing occurs, the absorbed power $\Pi_{a b s}$ and therefore the absorption coefficient can not be solely calculated from the surface impedance. Instead, the expression given by Eq. (37) has to be corrected for the power transmitted into the backing:

$$
\alpha_{\theta, \text { open }} \equiv \frac{\Pi_{\text {abs }}(\theta)}{\Pi_{\text {inc }}(\theta)}=\frac{4 \Re\left\{\tilde{Z}_{s}\right\}}{\left|\tilde{Z}_{s}+1\right|^{2}}-\left|\tau_{\theta}\right|^{2}
$$

where the transmission coefficient $\tau$ is defined as the fraction of the complex pressure amplitudes of the plane wave transmitted into the backing and the incident wave $\check{p}_{0}$.

For curtains that are not placed in the vicinity of a room surface, or generally for objects located in the diffuse field, the definition of a statistical absorption coefficient is ambiguous. Analogous to Eq. (39) we use the following definition

$$
\begin{aligned}
\alpha_{s, \text { open }} & =\frac{\int_{0}^{\pi} \Pi_{\mathrm{abs}}(\theta) \sin \theta d \theta}{\int_{0}^{\pi} \Pi_{\mathrm{inc}}(\theta) \sin \theta d \theta} \\
& =\int_{0}^{\pi} \alpha_{\theta, \text { open }}|\cos \theta| \sin \theta d \theta .
\end{aligned}
$$

where in order to derive Eq. (43), uniform sound intensity distribution and an infinitely extended surface is assumed, i.e. edge effects are not taken into account. Eq. (43) therefore corresponds to Paris' formula. However, a clear concept is the equivalent sound absorption area, which by relying on the above definition, can be formulated as 


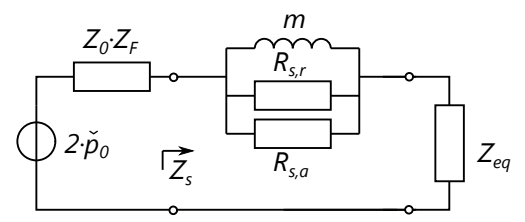

Figure 8: Equivalent electrical circuit of a single fabric sheet with a representative impedance $Z_{\text {eq }}$ for the field behind the sheet.

$$
A_{\text {open }}=2 S \cdot \alpha_{s, \text { open }}
$$

where $S$ denotes the one-sided surface area of the absorber. The factor 2 in Eq. (44) accounts for the effect that by placing the absorber in the diffuse field, the total intensity on the absorber surface, or in other words the effective area of the absorber, is doubled.

One has to note that when applying Eq. (37), (39) or (42) it is of utmost importance to use an extended reaction model for the air cavities as stated in section 2.6. By applying a local reaction assumption to thin absorbers with a backing air gap, significant errors of the absorption coefficient at oblique and random incidence may emerge [46].

\subsection{Single sheet case}

In this section the special case with only one single fabric $(N=1)$ is treated. The equivalent circuit is depicted in Fig. 8 with an impedance $Z_{\text {eq }}$ representing the surface impedance of the field behind the sheet. The surface impedance of the structure hence reduces to

$$
Z_{s}=Z_{f}+Z_{\text {eq }}
$$

For the rigidly terminated air cavity the impedance $Z_{\text {eq }}$ is given by Eq. (35). Eq. (45) can now be inserted into Eq. (37) to obtain the angle-dependent absorption coefficient of a single fabric backed by a rigidly terminated air cavity

$$
\alpha_{\theta}=\frac{4 \Re\left\{\tilde{Z}_{f}\right\}}{\left|\tilde{Z}_{f}-j \cot \left(k_{0} \cos \theta d\right)+1\right|^{2}}
$$

with 


$$
\tilde{Z}_{f}=\frac{Z_{f}}{Z_{0} / \cos \theta} .
$$

For the case without backing, i.e. air, $Z_{\mathrm{eq}}$ is given by Eq. (36). The surface impedance is thus given by $Z_{s}=Z_{f}+Z_{0} / \cos \theta$. By applying the potential divider rule to the equivalent circuit, the transmission coefficient can be found as

$$
\tau_{\theta}=\frac{2}{\tilde{Z}_{f}+2} .
$$

By inserting the surface impedance and Eq. (48) into Eq. (42) one obtains the oblique incidence absorption coefficient of a single fabric sheet without backing

$$
\alpha_{\theta, \text { open }}=\frac{4 \Re\left\{\tilde{Z}_{f}\right\}}{\left|\tilde{Z}_{f}+2\right|^{2}} .
$$

For the equivalent sound absorption area, inserting Eq. (49) into Eq. (43) and further into Eq. (44) and usage of symmetry yields

$$
A_{\text {open }}=16 S \cdot \int_{0}^{\pi / 2} \frac{\Re\left\{\tilde{Z}_{f}\right\}}{\left|\tilde{Z}_{f}+2\right|^{2}} \cos \theta \sin \theta d \theta
$$

\section{Validation}

In order to validate the model described in section 2, measurements on woven fabrics consisting of different synthetic yarns have been performed and compared to predictions. Reliable acoustical data could only be obtained by measurements with an impedance tube, i.e. under normal sound incidence.

\subsection{Measurement description}

Normal incidence absorption coefficients of fabrics were measured according to the standard ISO 10534-2[47], using a Brüel \& Kjaer two-microphone impedance tube of type 4206. The tube had a diameter of $10 \mathrm{~cm}$ allowing measurements in the range from $100 \mathrm{~Hz}$ to $1.75 \mathrm{kHz}$ with a frequency resolution of $5 \mathrm{~Hz}$. To minimize the influence of mounting and to reduce the 


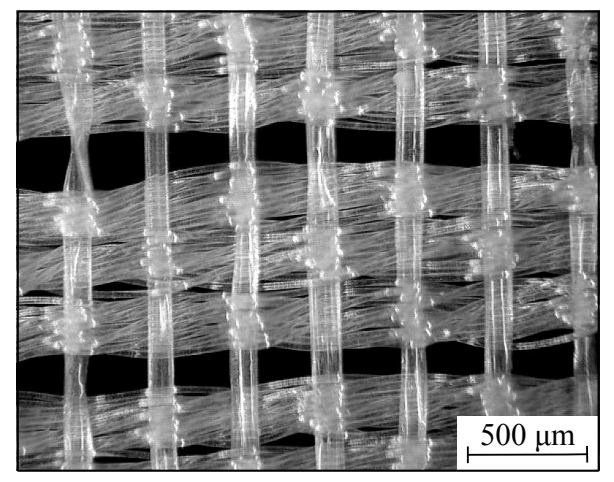

Figure 9: Macro photograph of woven fabric with rectangular interyarn pores (black areas) with hydraulic radius $\bar{r}=90 \mu \mathrm{m}$, interyarn porosity $\phi=10 \%$ and $\beta=50 \%$.

basic absorption of the empty tube, an additional ring was used which did not alter the diameter inside the tube. However, it has to be noted that the mounting prevents the fabric from freely moving and therefore the mechanical impedance of the membrane is altered. For each fabric, measurements were performed for two air cavity sizes, $d$, of 10 and $15 \mathrm{~cm}$.

In addition, geometrical parameters of each fabric were extracted from macro photographs, as shown in Fig. 9. A representative section of each fabric was chosen in which the average pore length and width, $a$ and $b$, were manually measured. Within the identical section also the open area (i.e. the sum of the interyarn pores) and the area covered by porous yarns were measured. From these values the hydraulic radius $\bar{r}$, the interyarn porosity, $\phi$, and the portion of the surface area with exposable porous yarns $\beta$ were calculated. Table 1 contains the geometrical data interyarn porosity $\phi$, the pore aspect ratio $a / b$, the hydraulic radius $\bar{r}$ and the portion of porous yarns $\beta$ of the 24 tested fabrics. For these geometrical parameters also the numerical value of the combined standard uncertainty, $u_{\mathrm{c}}$, in the measurement is stated. They were calculated according to the "Guide to the expression of uncertainty in measurement (GUM)" [48] using the law of propagation of uncertainty and can be further used to estimate the uncertainty of the prediction [49]. The surface mass density, $m$, of each fabric was measured by a precision scale. Table 1 also contains the specific airflow resistance $R_{s}$ which was obtained from the impedance tube measurements by a curve fitting procedure [6]. 


\begin{tabular}{cccccccc}
$\begin{array}{c}\text { Fabric } \\
\text { ID \# }\end{array}$ & $\phi$ & $\bar{r}$ & $a / b$ & $\beta$ & $R_{s}$ & $m$ & $f_{c}$ \\
{$[\%]$} & {$[\mu \mathrm{m}]$} & {$[-]$} & {$[\%]$} & $\mathrm{Pa} \mathrm{s} / \mathrm{m}]$ & {$\left[\mathrm{g} / \mathrm{m}^{2}\right]$} & {$[\mathrm{Hz}]$} \\
\hline 1 & $38(4)$ & $61(1)$ & $1.2(0.0)$ & $11(2)$ & 10 & 36 & 44 \\
2 & $31(5)$ & $53(2)$ & $1.6(0.1)$ & $0(0)$ & 11 & 46 & 38 \\
3 & $25(4)$ & $39(2)$ & $1.7(0.1)$ & $33(6)$ & 18 & 39 & 73 \\
4 & $22(4)$ & $38(2)$ & $1.6(0.1)$ & $22(4)$ & 24 & 59 & 65 \\
5 & $13(3)$ & $51(3)$ & $1.9(0.1)$ & $28(4)$ & 19 & 55 & 55 \\
6 & $13(3)$ & $33(2)$ & $2.8(0.2)$ & $44(5)$ & 27 & 39 & 110 \\
7 & $13(3)$ & $51(3)$ & $1.6(0.1)$ & $18(3)$ & 25 & 40 & 99 \\
8 & $11(2)$ & $49(2)$ & $1.1(0.0)$ & $33(4)$ & 29 & 54 & 85 \\
9 & $11(3)$ & $33(2)$ & $1.2(0.1)$ & $59(14)$ & 41 & 64 & 102 \\
10 & $11(4)$ & $31(3)$ & $2.5(0.2)$ & $49(10)$ & 32 & 95 & 54 \\
11 & $9.1(2.3)$ & $41(3)$ & $6.2(0.5)$ & $0(0)$ & 36 & 58 & 99 \\
12 & $8.5(1.9)$ & $32(2)$ & $1.2(0.1)$ & $17(3)$ & 55 & 42 & 208 \\
13 & $7.5(1.7)$ & $40(2)$ & $1.9(0.1)$ & $11(2)$ & 52 & 52 & 159 \\
14 & $6.8(3.1)$ & $37(3)$ & $1.1(0.1)$ & $72(11)$ & 50 & 68 & 117 \\
15 & $5.9(1.0)$ & $27(1)$ & $1.4(0.1)$ & $41(4)$ & 85 & 70 & 193 \\
16 & $4.1(1.0)$ & $36(2)$ & $1.7(0.1)$ & $13(3)$ & 105 & 54 & 309 \\
17 & $4.0(0.9)$ & $34(2)$ & $1.2(0.1)$ & $28(6)$ & 105 & 272 & 61 \\
18 & $3.3(0.8)$ & $30(2)$ & $2.5(0.2)$ & $38(4)$ & 135 & 75 & 286 \\
19 & $2.8(1.1)$ & $35(4)$ & $6.9(0.8)$ & $18(1)$ & 122 & 79 & 246 \\
20 & $2.5(0.6)$ & $30(2)$ & $2.4(0.1)$ & $29(3)$ & 105 & 69 & 242 \\
21 & $2.1(0.6)$ & $27(2)$ & $1.2(0.1)$ & $4(2)$ & 165 & 47 & 559 \\
22 & $1.8(1.8)$ & $22(6)$ & $10.3(3.0)$ & $18(2)$ & 268 & 77 & 554 \\
23 & $1.5(1.1)$ & $25(5)$ & $9.9(2.0)$ & $18(2)$ & 208 & 79 & 419 \\
24 & $1.4(1.0)$ & $27(6)$ & $10.1(2.0)$ & $22(2)$ & 304 & 76 & 637
\end{tabular}

Table 1: Non-acoustical measurement data of 24 fabric types (identical fabric IDs as in [6] were used for replicability): Geometrical data obtained from macro photographs, indirectly measured specific airflow resistance, $R_{s}$, and surface mass density, $m$, and therefrom calculated cutoff frequency, $f_{c}$, according to Eq. (34). For the geometrical parameters also the combined standard uncertainty, $u_{\mathrm{c}}$, of the measurement is stated in brackets. 


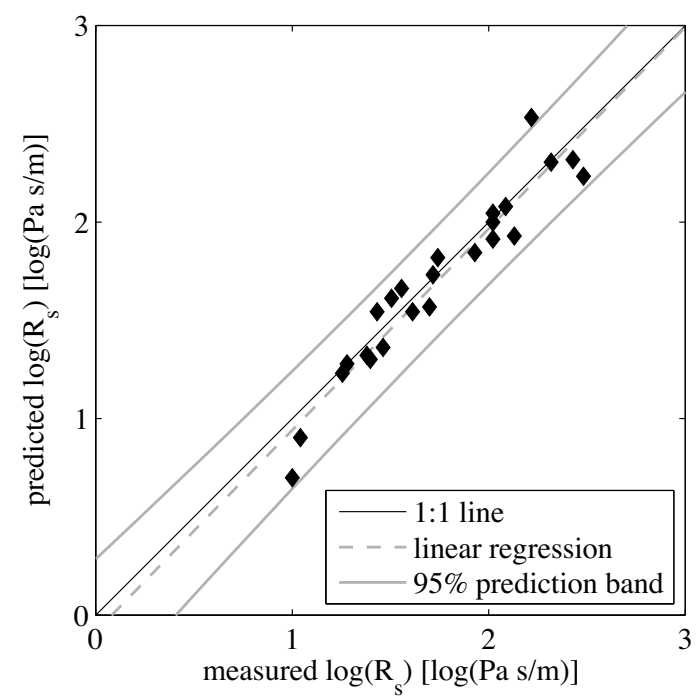

Figure 10: Comparison of measured and predicted specific airflow resistances of 24 woven fabrics (see Table 1). The straight black line is the identity line. The gray lines show the linear regression line $\left(R^{2}=0.92\right)$ of $\log \left(R_{s}\right)$ and its $95 \%$ prediction interval band for future estimates.

\subsection{Comparison of measured and predicted specific airflow resistances}

Before evaluating measured and predicted absorption coefficients, as a first validation step, indirectly measured and predicted specific airflow resistances, $R_{s}$, of the 24 woven fabrics were compared. The indirectly measured specific airflow resistances of the fabrics are listed in Table 1. They cover a wide range from 10 to $300 \mathrm{~Pa} \mathrm{~s} / \mathrm{m}$.

The specific airflow resistance of each fabric was predicted based on its geometrical parameters. At first, the specific airflow resistance corresponding to the interyarn airflow, $R_{s, r}$, was calculated using Eq. (21). The thereby used hydraulic radii, $\bar{r}$, and porosities, $\phi$, are given in in Table 1 . As no knowledge about the thicknesses of the fabrics, or to be more precise, the lengths of the pores, was available, for all calculations the parameter $h$ was set to $50 \mu \mathrm{m}$, which corresponds approximately to the diameter of one yarn. The geometrical tortuosity of the interyarn pores was set to $\alpha_{\infty}=1$ and the dynamic viscosity of air $\mu=18 \mu \mathrm{Pa}$ s. Then the specific airflow resistance corresponding to the intrayarn airflow, $R_{s, a}$ was calculated using Eq. (23). The measured portions, $\beta$, of the surface area with exposable porous yarns 
from Table 1 were used. However, the flow resistivity, $\sigma$, of the involved porous yarns, which is required in Eq. (23), is unknown. Therefore a rough estimate of $\sigma$ was used which was set globally, i.e. identical for all 24 fabrics, to $\sigma=10^{6} \mathrm{~Pa} \mathrm{~s} / \mathrm{m}^{2}$. Finally, the total static specific airflow resistance, $R_{s}$, was predicted by inserting the contributions $R_{s, r}$ and $R_{s, a}$ into Eq. (20).

Figure 10 shows the comparison of measured and predicted specific airflow resistances. Measured and predicted values agree well over a wide rangeacross which also the variance of the deviations stays constant. Thus, as both axes in Fig. 10 are plotted in logarithmic scale, it can be concluded that the relative prediction error of the model remains constant over the considered range of specific airflow resistances and fabric types. Fig. 10 also contains the linear regression line $\left(R^{2}=0.92\right)$ of $\log \left(R_{s}\right)$ and its $95 \%$ prediction interval band for future estimates. In the following sections the prediction of absorption coefficients are evaluated.

\subsection{Comparison of measured and predicted normal incidence absorption curves}

The calculations of normal incidence absorption coefficients were performed by use of the equations given in section 2.5 and Eq. (46). In a first step the specific airflow resistance of each fabric was estimated as elucidated in the previous section. To do this, the measured geometrical fabric parameters hydraulic radius, $\bar{r}$, porosity, $\phi$, and the ratio $\beta$ (see Table 1 ) were used. The parameters $\mu, h, \alpha_{\infty}$ and $\sigma$ were set globally as in the previous section. Then the fabric impedance was approximated by Eq. (32) by use of the measured surface mass density (see Table 1). The use of Eq. (32) induces that the flexural rigidity $\left(D^{\prime}=0 \mathrm{~Pa} \mathrm{~s} / \mathrm{m}\right)$ and acoustical reactance $\left(Z_{\mathrm{ac}}=R_{s}\right)$ are neglected. In a last step, the obtained fabric impedance was inserted into Eq. (46) where the distance $d$ was set as in the impedance tube measurement, i.e. either 10 or $15 \mathrm{~cm}$. In Eq. (46) the angle of incidence, $\theta$, was set to 0 and $\rho_{0}=1.2 \mathrm{~kg} / \mathrm{m}^{3}$ and $c_{0}=343 \mathrm{~m} / \mathrm{s}$.

Fig. 11 exemplarily compares measured and predicted normal incidence absorption coefficients of three fabrics (see Table 1) for two air cavity sizes each. Generally, the frequency dependency of measured and calculated curves coincide very well. Deviations at low frequencies can be explained by acoustical measurement uncertainties and possibly by the influence of a mechanical resistance which was neglected in the calculation. Deviations at the local minima of the absorption curves, at 1.7 and $1.2 \mathrm{kHz}$, respectively, are most likely due to the residual absorption of the measurement system including dissipation effects inside the air cavity. Besides these deviations the largest 
ID
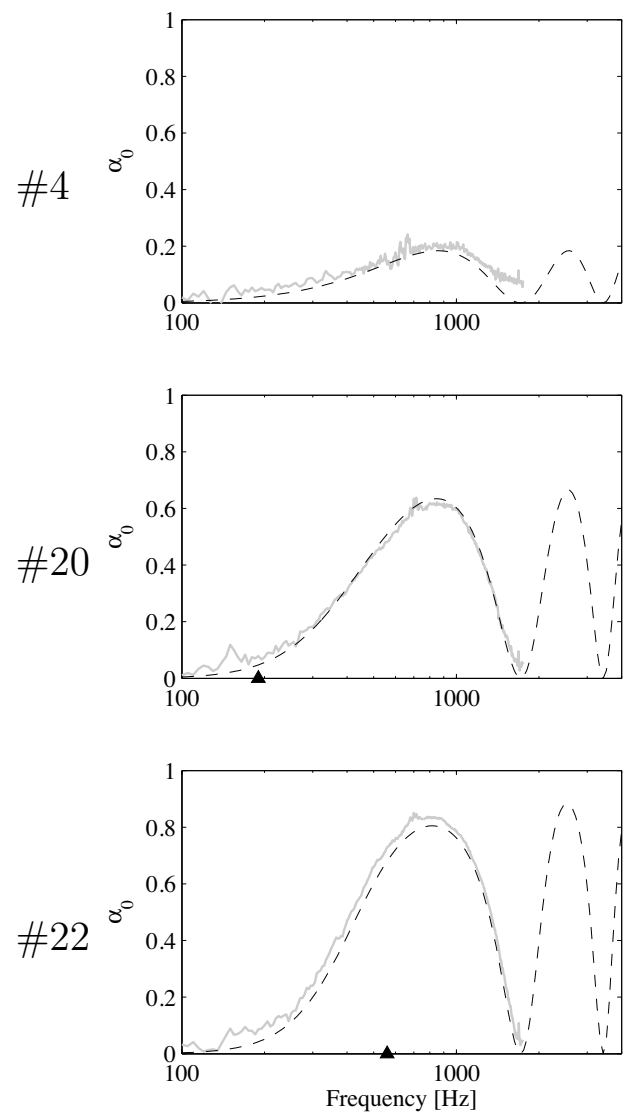

$d=15 \mathrm{~cm}$
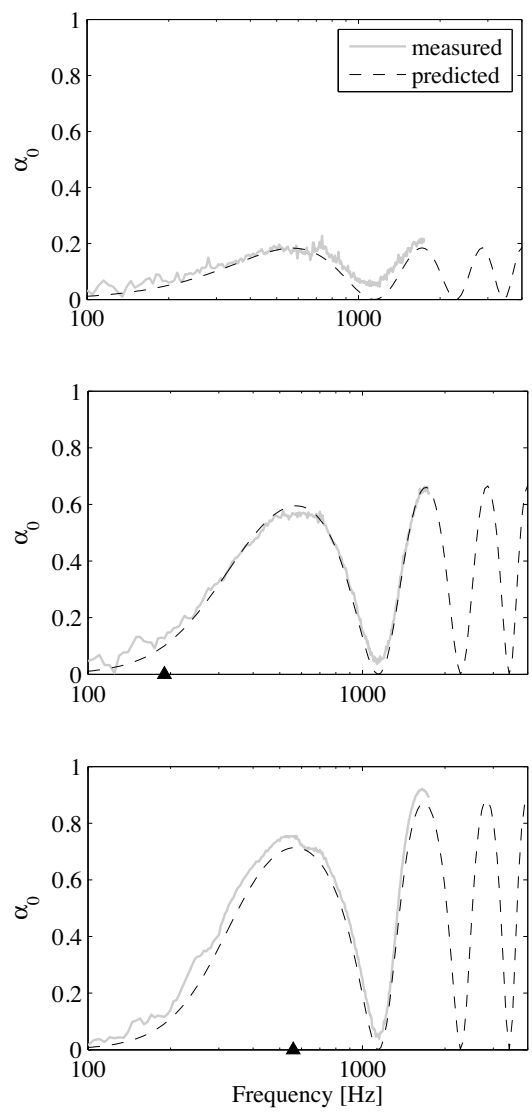

Figure 11: Measured (grey) and predicted (black) normal incidence absorption coefficients of the three fabrics \#4, \#20 and \#22 (see Table 1) with two air cavity sizes $d=10 \mathrm{~cm}$ (left column) and $d=15 \mathrm{~cm}$ (right column). The triangle on the abscissa indicates the cutoff frequency $f_{c}$ (Eq. (34)). 
differences between measurement and prediction are observed at the local maxima of the curves. The local maxima occur at frequencies where the imaginary part of the surface impedance, $Z_{s}$, vanishes. To a first approximation this condition is fullfilled for frequencies where $d \approx n \lambda / 4$ with $n$ an odd integer. In the following section, only absorption coefficients at these frequencies are considered.

In Fig. 11 the effect of sound induced vibrations can be well observedespecially for fabric \#22, which has a cutoff frequency above $500 \mathrm{~Hz}$, in the case of $d=15 \mathrm{~cm}$ (bottom right plot). There the first local maximum (at $570 \mathrm{~Hz}$ ) is significantly lowered compared to the second local maximum (at $1.7 \mathrm{kHz}$ ) due to the motion of the fabric. This effect is investigated further in section 4.1 .

\subsection{Validation of the geometry-based model to predict the normal incidence absorption coefficient}

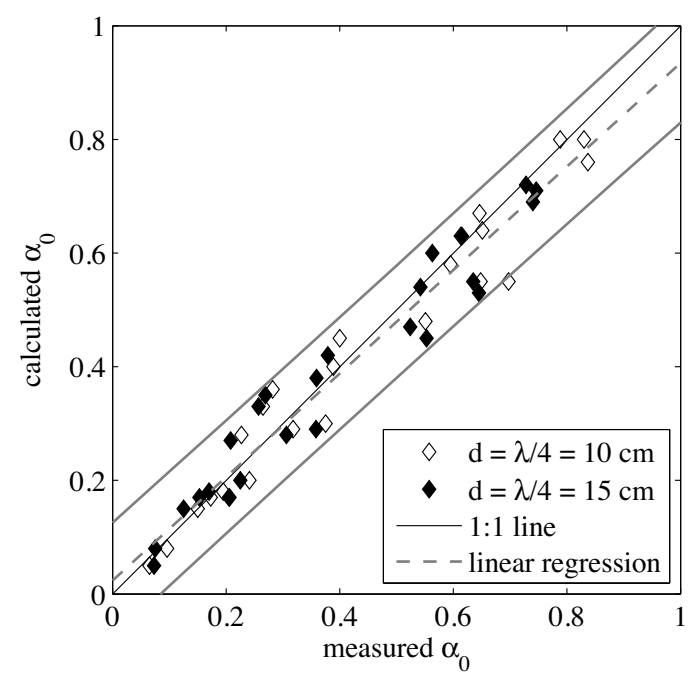

Figure 12: Comparison of measured and predicted normal incidence absorption coefficients at $\mathrm{d}=\lambda / 4=10 \mathrm{~cm}$ (white) and $15 \mathrm{~cm}$ (black) of 24 woven fabrics (see Table 1). The straight black line is the identity line. The gray lines shows the linear regression line $\left(R^{2}=0.95\right)$ and the $95 \%$ prediction interval band for future estimates.

Since the largest differences between measurement and prediction are expected at the local maxima of the absorption coefficient curves, as a worst 
case consideration, measured and calculated normal incidence absorption coefficients at a quarter wavelength distance to the wall are compared.

In order to validate the geometry-based model, the set of 24 different fabrics according to Table 1 was used. The surface mass density, $m$, varied from 36 to $270 \mathrm{~g} / \mathrm{m}^{2}$ whereas all fabrics but one were lighter than $100 \mathrm{~g} / \mathrm{m}^{2}$. The interyarn porosity, $\phi$, varied from 1.4 to $38 \%$; the hydraulic radius, $\bar{r}$, from 22 to $61 \mu \mathrm{m}$, the pore aspect ratio $a / b$ from 1.1 to 10 and the amount, $\beta$, of exposable porous yarns from 0 to $70 \%$. Also the indirectly measured specific airflow resistance covered a large region ranging from 10 to $300 \mathrm{~Pa} \mathrm{~s} / \mathrm{m}$. Nine fabrics had a cutoff frequency $f_{c}$ above $200 \mathrm{~Hz}$. The maximum cutoff frequency lied above $600 \mathrm{~Hz}$. For the calculation, the identical parameters as in the previous section have been used.

Fig. 12 shows the comparison of 24 fabrics for two air cavity sizes. Measured and estimated absorption coefficients exhibit excellent agreement, with mean value and standard deviation of the differences of $0.01 \pm 0.05$. The mean value does not significantly differ from zero with a probability of 0.90 (onesample two-tailed t-test). The gray lines in Fig. 12 show the linear regression line and the $95 \%$ prediction interval band for future estimates of the absorption coefficient by the proposed model and measurement techniques employed. The prediction errors are astonishing small in the light of the uncertainties of the geometrical input parameters, i.e. uncertainties due to local variations inside the fabric, as can be seen in Fig. 9, and the measurement uncertainties given in Table 1. It can be concluded that-within the tested set of fabrics - the model covers the predominant effects for the prediction of the considered absorption coefficients.

Compared to predictions by a former, simpler model [6], the standard deviation of the errors could be reduced by a factor of 2 . The reason for this improvement lies in the consideration of the intrayarn airflow and the usage of the hydraulic pore radius for the interyarn airflow, instead of the area equivalent pore radius. The following section further investigates the first part of this explanation.

\subsection{Influence of interyarn and intrayarn airflow contribution on absorption coefficient}

In this section, the respective contributions of the intrayarn and the interyarn airflow on the absorption coefficient are investigated. This type of analysis can be especially beneficial in the acoustical design or optimisation process of fabrics. There it is important to assess the effect of the fabric 


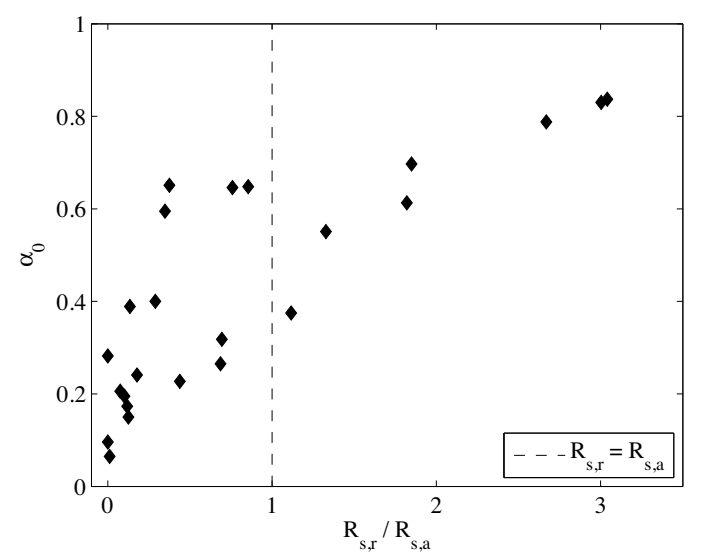

Figure 13: Measured normal incidence absorption coefficients at $d=\lambda / 4=10 \mathrm{~cm}$ of 24 woven fabrics (see Table 1) as a function of the theoretically obtained ratio of interyarn and intrayarn specific airflow resistance.

texture or the textile microstructure, i.e. the weaving pattern, type of yarns, etc. For the set of 24 fabrics (see Table 1) the ratio of the interyarn and intrayarn specific airflow resistance $R_{s, r} / R_{s, a}$, both calculated as in the previous sections, was determined. In Fig. 13 for each fabric type, the normal incidence absorption coefficient at $d=\lambda / 4=10 \mathrm{~cm}$ is plotted against this ratio. The set of considered fabrics covers a wide range of this ratio from 0 to 3. Fig. 13 reveals that for the fabrics with low absorption coefficients, the total airflow through the fabric is dominated by the interyarn airflow. However, absorption coefficients above 0.5 can be realized either by dominating interyarn or intrayarn airflow. For the most effective sound absorbing fabrics in this set, the intrayarn airflow dominates the total airflow. These observations demonstrate that it is crucial to consider the interyarn as well as the intrayarn airflow in the model.

\section{Application}

In this section, with the help of the model presented in section 2, four questions arising from practical applications of curtains as sound absorbers shall be treated and answered:

1. Is there a relevant influence of the finite mass of a lightweight curtain on its absorption properties? (see section 4.1) 

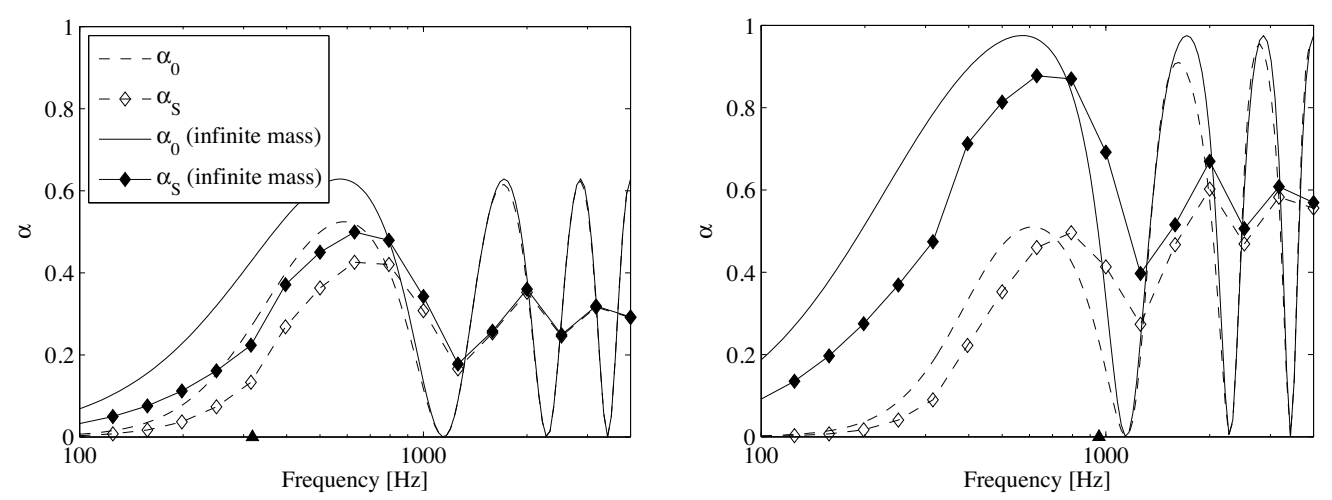

Figure 14: Calculated absorption coefficients of two fabrics at $d=15 \mathrm{~cm}$ distance to the wall. Both fabrics have a surface mass density of $50 \mathrm{~g} / \mathrm{m}^{2}$ but a different specific airflow resistance of $100 \mathrm{~Pa} \mathrm{~s} / \mathrm{m}$ (left) and $300 \mathrm{~Pa} \mathrm{~s} / \mathrm{m}$ (right). The cutoff frequency $f_{c}$ is indicated with a triangle on the abscissa. The solid lines show calculations for an infinite mass.

2. What is the optimal specific airflow resistance of a curtain fabric? (see section 4.2)

3. How does a curtain in the center of a room perform compared to the conventional mounting, in the vicinity of a room surface? (see section 4.3)

4. Is there an acoustical benefit in using multiple fabric sheets for a curtain system? (see section 4.4)

\subsection{Influence of finite mass}

With the model described in section 2 , the effect of the finite mass on the absorption properties of lightweight curtains can be investigated. Fig. 14 exemplarily shows calculated values for two lightweight single-sheet curtains. Both fabrics have a surface mass density of $50 \mathrm{~g} / \mathrm{m}^{2}$ but differ in terms of their specific airflow resistance which is $100 \mathrm{~Pa} \mathrm{~s} / \mathrm{m}$ (left) and $300 \mathrm{~Pa} \mathrm{~s} / \mathrm{m}$ (right), respectively. Therefore also their cutoff frequencies are different, i.e. 320 and $950 \mathrm{~Hz}$. In addition to the calculations by the proposed model also calculations with the assumption of an infinite mass have been performed and are shown as solid lines in Fig. 14 for comparison. From Fig. 14 it becomes clear that the normal incidence absorption coefficient as well as the statistical absorption coefficient can be drastically overestimated over a broad frequency range if not taking into account the finite mass of the fabric. Thus the vibration of a fabric is an important aspect in the design of lightweight curtains. 


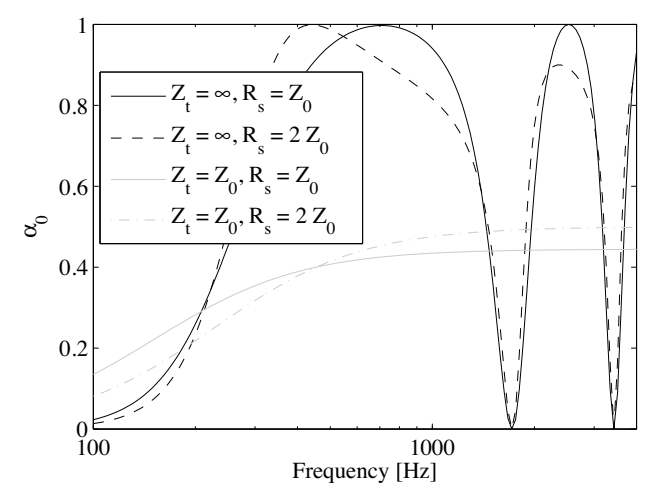

Figure 15: Calculated normal incidence absorption coefficients of two fabrics with identical surface mass density $m=280 \mathrm{~g} / \mathrm{m}^{2}$ but different specific airflow resistance of $Z_{0}$ and $2 Z_{0}$, respectively. Both fabrics are backed with a rigidly terminated air cavity of size $d=10 \mathrm{~cm}$ on the one hand $\left(Z_{t}=\infty\right)$ and with free field on the other hand $\left(Z_{t}=Z_{0}\right)$.

\subsection{Optimal specific airflow resistance}

For some special cases the optimal specific airflow resistance of a sheet can be given analytically in order to reach maximal absorption. For a single sheet in front of a rigidly backed air cavity, the optimal specific airflow resistance can be found by inserting Eq. (32) into Eq. (46) and differentiating with respect to $R_{s}$. For normal sound incidence and quarter wavelength distance to the wall follows $[6]$

$$
R_{s, \mathrm{opt}, \mathrm{close}}=\frac{Z_{0}}{\sqrt{1+\left(\frac{2 \rho_{0} d}{\pi m}\right)^{2}}} .
$$

Eq. (51) implies that for large surface mass densities, small distances to the wall and thus high frequencies, the optimal specific airflow resistance equals the characteristic impedance of air $Z_{0}$ (see Fig. 15). However, for smaller surface mass densities or larger cavity sizes, i.e. lower frequencies, the optimal specific airflow resistance gets smaller. By inserting Eq. (51) into Eq. (32) and further into Eq. (46) one finds that at quarter wavelength distance to the wall the maximal possible absorption coefficient of 1 is only reached for $m \rightarrow \infty$, i.e. for $R_{s, \text { opt,close }}=Z_{0}$. Note that this limitation only applies at quarter wavelength distance to the wall-e.g. Fig. 15 shows that also fabrics with $R_{s}>Z_{0}$ can exhibit large normal incidence absorption coefficients at certain frequencies. 
For a freely hanging fabric sheet the optimal specific airflow resistance for maximal $\alpha_{\theta}$ can be obtained by inserting Eq. (32) into Eq. (49) and some calculation $[9]$ :

$$
R_{s, \text { opt }, \text { open }}=\frac{1}{\sqrt{\left(\frac{1}{\omega m}\right)^{2}+\left(\frac{\cos \theta}{2 Z_{0}}\right)^{2}}}
$$

It can be seen that the optimal specific airflow resistance does not only depend on the surface mass density and frequency but also on the angle of the incident wave. For $\omega m \rightarrow \infty$ and normal sound incidence, $R_{s, \text { opt,open }}=2 Z_{0}$. The absorption coefficient in this case is shown in Fig. 15. This implies that the optimal specific airflow resistance also strongly depends on the backing. By inserting Eq. (52) into Eq. (32) and further into Eq. (49) it follows that the oblique incidence absorption coefficient, $\alpha_{\theta}$, of a freely hanging fabric sheet never exceeds 0.5 [9]. For $\omega m \rightarrow \infty$ this value is reached if the power of the reflected wave equals the power of the transmitted wave.

For practical and more complex cases, i.e. when maximizing the statistical absorption coefficient in a certain frequency range or for multilayer curtains, an optimisation procedure with an appropriate optimisation criterion has to be performed.

\subsection{Freely hanging curtains}

It is usually assumed that the sound absorber is placed at or close to a room surface. However, especially for curtains, which can be easily mounted hanging freely in the room, it is interesting to compare the absorptive effectiveness to the usual case, i.e. mounted with a rigidly backed air cavity of defined extent. To do this, as pointed out in section 2.7, the equivalent sound absorption area $A$ shall be considered. This quantity is necessary to predict the reverberation time of a room by Sabine's formula.

In the following we compare the equivalent sound absorption area of a certain piece of fabric which is either mounted at a defined distance to a hard backing or placed in the diffuse field, i.e. freely hanging. For a single fabric sheet $A_{\text {open }}$ is calculated by Eq. (50). As in Eq. (50) edge effects are neglected, also in the calculation of $A$ for the rigidly backed curtain by Eq. (41), edge effects are neglected, i.e. $Z_{F}=1 / \cos \theta$ in Eq. (39). However, the angle weighting function $w(\theta)$ according to Eq. (40) accounting for nonuniform intensity distribution on room surfaces is used. The surface area of both absorbers is arbitrarily set to $S=1 \mathrm{~m}^{2}$. The incident angle $\theta$ was 

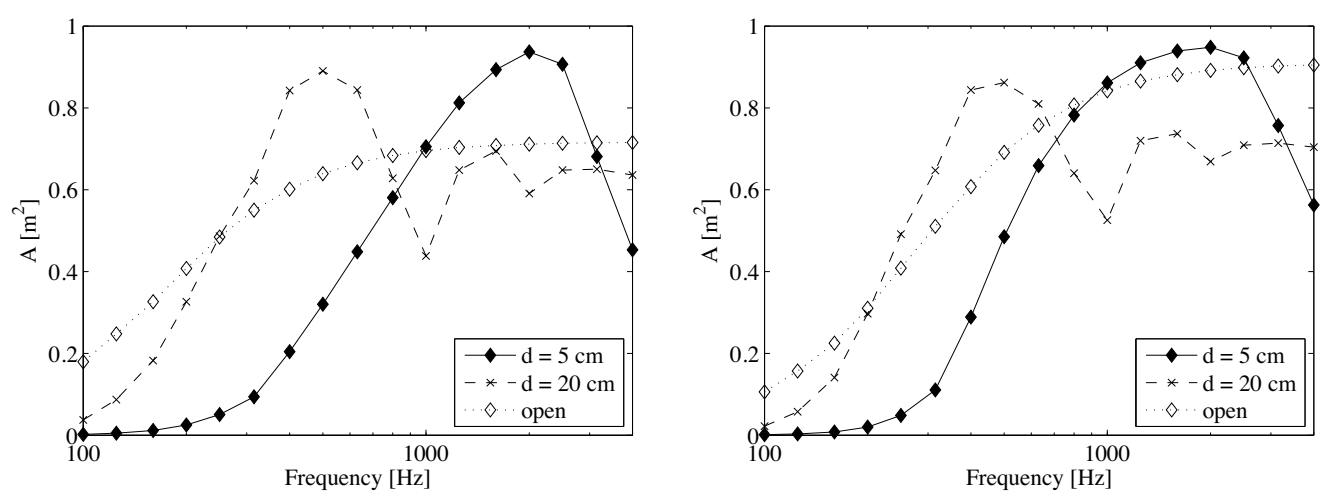

Figure 16: Comparison of calculated equivalent absorption areas of single-sheet fabrics with different backings. The fabrics from Fig. 15 are shown, i.e. $m=280 \mathrm{~g} / \mathrm{m}^{2}$ but different specific airflow resistance of $Z_{0}$ (left) and $2 Z_{0}$ (right), respectively. For both fabrics, the cases with air cavities of $5 \mathrm{~cm}$ and $20 \mathrm{~cm}$, respectively, and the freely hanging case are shown.

discretized in steps of $2^{\circ}$. Calculations were performed for $1 / 27$ octave band center frequencies and the resulting equivalent sound absorption areas were averaged in order to obtain values for $1 / 3$ octave bands.

Fig. 16 compares calculated equivalent absorption areas of single-sheet curtains consisting of the fabrics from Fig. 15. Fig. 16 reveals that the same fabric sheet placed in the diffuse field of a room, instead of placing it at a certain distance to the wall, is capable to absorb sound energy in the same order of magnitude - indeed with a much smoother frequency dependency because no air cavity resonances exist. At low frequencies the absorptive effectiveness of the freely hanging fabric is even higher than if mounted with a rigidly backed air cavity.

\subsection{Benefit of multiple fabric sheets}

The question rises weather there is an advantage of having multiple fabric sheets in a curtains system - as it is the case for MPPs. To answer this question, a parametric study was conducted. Initially, a proper optimisation criterion reflecting the specific needs is required. If looking at typical statistical sound absorption coefficients in $1 / 3$ octave bands (e.g. Fig. 14) it can be observed that they exhibit a dip slightly above half wavelength distance to the wall. If focusing on high broadband absorption the size of this dip determines the absorptive quality of the absorber. In order to easily quantify 
the sound absorption potential of a curtain, a single-valued minimal absorption coefficient, $\alpha_{\min }$, was defined. $\alpha_{\min }$ is the minimal value of the statistical absorption coefficients for the $1 / 3$ octave bands bands $500 \mathrm{~Hz}$ to $5 \mathrm{kHz}$ :

$$
\alpha_{\min }=\min \left(\alpha_{s, f}\right) \text { for } 500 \mathrm{~Hz} \leq f \leq 5 \mathrm{kHz} .
$$

For a fair comparison between curtain layouts with varying number of sheets it was decided to keep constant the total extent of the absorber

$$
d=\sum_{i=1}^{N} d_{i}
$$

and the total surface mass density

$$
m=\sum_{i=1}^{N} m_{i} .
$$

Under these conditions, the absorption coefficient, $\alpha_{\min }$, is maximized by systematic variation of the specific airflow resistances $R_{s, i}$, the surface mass densities $m_{i}$ and the air cavity sizes $d_{i}$.

For the numerical simulations the model described in section 2 was used. The fabrics were characterized by their surface mass density and specific airflow resistance. Eq. (30) and Eq. (39) were used to obtain the statistical absorption coefficient. The weighting function according to Jeong (Eq. (40)) was adopted. Edge effects were not taken into account, i.e. $Z_{F}=1 / \cos \theta$. The calculations were performed in $1 / 27$ octave bands which were then averaged to obtain statistical absorption coefficients for the $1 / 3$ octave bands from $500 \mathrm{~Hz}$ to $5 \mathrm{kHz}$. For $\theta$ a resolution of $2^{\circ}$ was used. Speed of sound $c_{0}$ and air density $\rho_{0}$ were set to $343 \mathrm{~m} / \mathrm{s}$ and $1.2 \mathrm{~kg} / \mathrm{m}^{3}$, respectively.

Fig. 17 shows the minimal absorption coefficients $\alpha_{\min }$ of optimised curtains consisting of one (right) and two (left) fabric sheets with a rigidly backed air cavity. Values are shown for total surface mass densities, $m$, ranging from 0 to $400 \mathrm{~g} / \mathrm{m}^{2}$ and total absorber extents, $d$, from 0 to $30 \mathrm{~cm}$. The discretization of these parameters as well as the individual surface mass densities, $m_{i}$, and air cavity sizes, $d_{i}$, of the double sheet curtain were $20 \mathrm{~g} / \mathrm{m}^{2}$ and $2 \mathrm{~cm}$, respectively. The specific airflow resistance of each fabric was systematically varied by setting $R_{s, i}=2^{k_{i} / 3} \mathrm{~Pa} \mathrm{~s} / \mathrm{m}$ with integers $20 \leq k_{i} \leq 30$, i.e. logarithmically spaced between 100 and $1000 \mathrm{~Pa} \mathrm{~s} / \mathrm{m}$. 

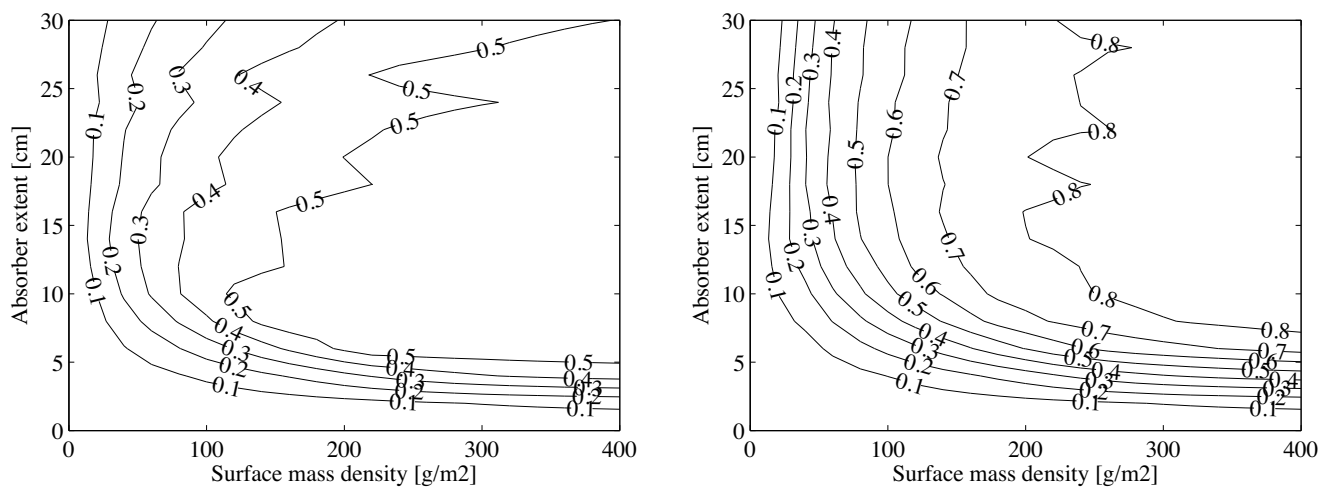

Figure 17: Minimal absorption coefficients $\alpha_{\min }$ of optimised curtains consisting of one (left) and two (right) fabric sheets with a hard backing, as a function of total surface mass density and the absorber thickness.

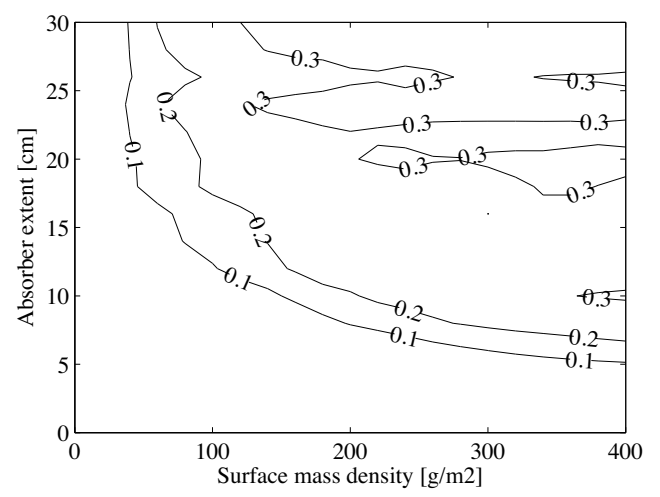

Figure 18: Absorption coefficient differences $\Delta \alpha_{\text {min }}$ of optimised double fabric and single fabric curtain with hard backing.

For the single and the double sheet curtain, Fig. 17 reveals that a minimal total surface mass density and a minimal absorber extent is required to obtain a substantial minimal absorption coefficient. Both curtain systems feature a saturation region which in the double sheet case, however, has significantly larger values compared to the single sheet curtain. The peak values are 0.59 for the single sheet and 0.88 for the double sheet curtain, respectively.

Fig. 18 shows the difference plot of the data from Fig. 17. It can be interpreted as the benefit of an optimised double sheet curtain compared to an optimised single sheet curtain. A reasonable benefit can only be gained if the extent of the absorber and the total surface mass density have a certain 

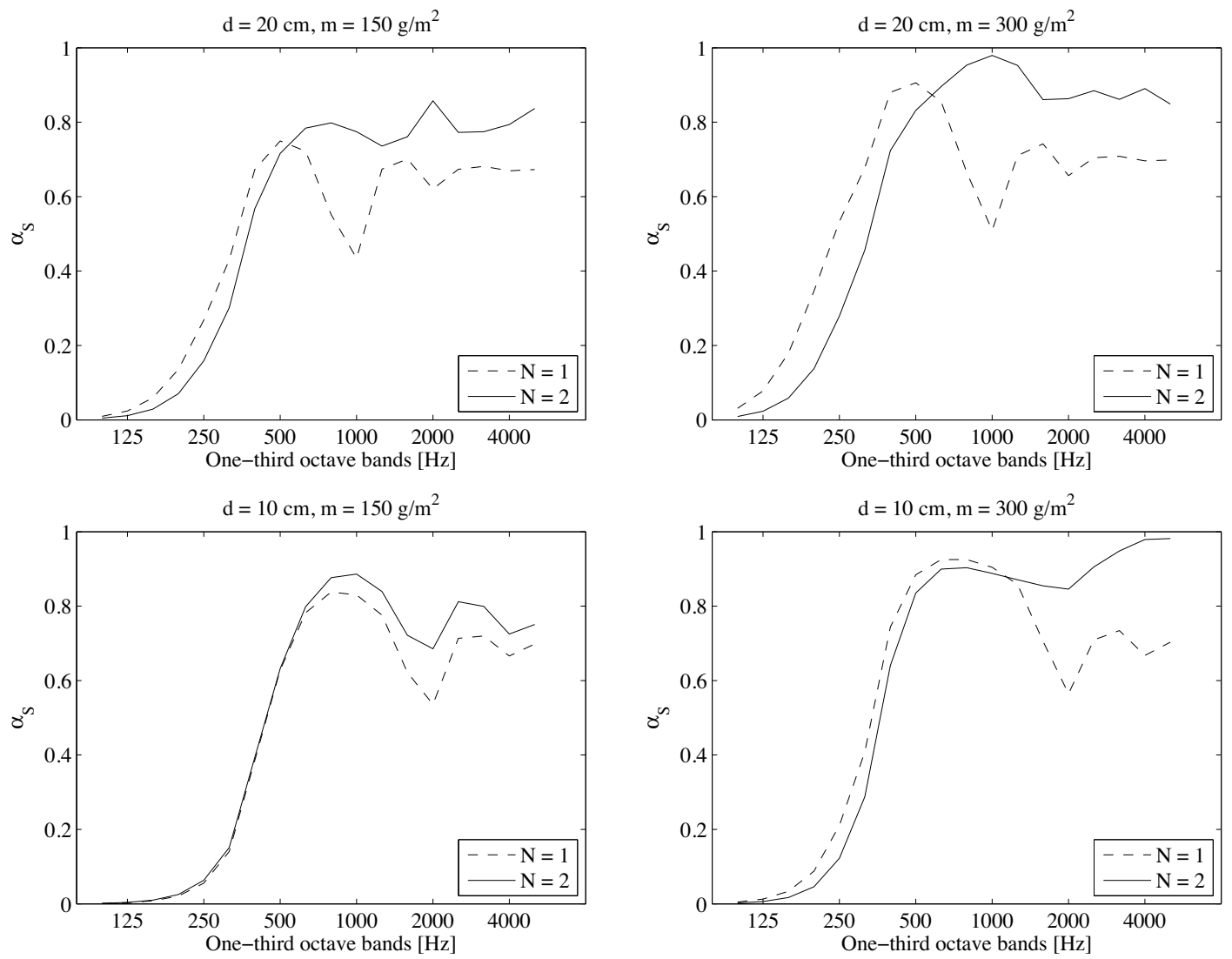

Figure 19: Comparison of statistical absorption coefficients of optimised single $(N=1)$ and double $(N=2)$ fabric curtains with a hard backing, for $d=\{10,20\} \mathrm{cm}$ and $m=$ $\{150,300\} \mathrm{g} / \mathrm{m}^{2}$. 


\begin{tabular}{l|c|c|c|c|} 
& \multicolumn{4}{|c|}{$d=10 \mathrm{~cm}$} \\
& $m=150 \mathrm{~g} / \mathrm{m}^{2}$ & $m=300 \mathrm{~g} / \mathrm{m}^{2}$ \\
$N$ & 1 & 2 & 1 & 2 \\
\hline$R_{s, 1}[\mathrm{~Pa} \mathrm{~s} / \mathrm{m}]$ & 1020 & 650 & 1020 & 510 \\
$m_{1}\left[\mathrm{~g} / \mathrm{m}^{2}\right]$ & 150 & 130 & 300 & 60 \\
$d_{1}[\mathrm{~cm}]$ & 10 & 4 & 10 & 2 \\
$R_{s, 2}[\mathrm{~Pa} \mathrm{~s} / \mathrm{m}]$ & - & 100 & - & 1020 \\
$m_{2}\left[\mathrm{~g} / \mathrm{m}^{2}\right]$ & - & 20 & - & 240 \\
$d_{2}[\mathrm{~cm}]$ & - & 6 & - & 8 \\
\hline$\alpha_{\min }$ & 0.54 & 0.63 & 0.56 & 0.84
\end{tabular}

Table 2: Comparison of optimised cases for $d=10 \mathrm{~cm}$

\begin{tabular}{l|c|c|c|c|} 
& \multicolumn{4}{|c|}{$d=20 \mathrm{~cm}$} \\
$N$ & $m=150 \mathrm{~g} / \mathrm{m}^{2}$ & $m=300 \mathrm{~g} / \mathrm{m}^{2}$ \\
$N$ & 1 & 2 & 1 & 2 \\
\hline$R_{s, 1}[\mathrm{~Pa} \mathrm{~s} / \mathrm{m}]$ & 510 & 260 & 650 & 410 \\
$m_{1}\left[\mathrm{~g} / \mathrm{m}^{2}\right]$ & 150 & 30 & 300 & 120 \\
$d_{1}[\mathrm{~cm}]$ & 20 & 6 & 20 & 12 \\
$R_{s, 2}[\mathrm{~Pa} \mathrm{~s} / \mathrm{m}]$ & - & 410 & - & 810 \\
$m_{2}\left[\mathrm{~g} / \mathrm{m}^{2}\right]$ & - & 120 & - & 180 \\
$d_{2}[\mathrm{~cm}]$ & - & 14 & - & 8 \\
\hline$\alpha_{\min }$ & 0.43 & 0.72 & 0.51 & 0.83
\end{tabular}

Table 3: Comparison of optimised cases for $d=20 \mathrm{~cm}$

magnitude. It can be concluded that a double sheet curtains performs superiorly to a single sheet curtain if the total extent is larger than $10 \mathrm{~cm}$ and the surface mass density lies above $100 \mathrm{~g} / \mathrm{m}^{2}$.

In Fig. 19 examples of statistical absorption coefficients of optimised single $(N=1)$ and double $(N=2)$ fabric cases are shown. The curves represent the optimised solutions for all combinations of input parameters $d=\{10,20\} \mathrm{cm}$ and $m=\{150,300\} \mathrm{g} / \mathrm{m}^{2}$. In all four situations a clear improvement of the absorptive behavior at mid and high frequencies can be observed. The lowest effect is observed at $d=10 \mathrm{~cm}$ and $m=150 \mathrm{~g} / \mathrm{m}^{2}$; the highest at $d=20 \mathrm{~cm}$ and $m=300 \mathrm{~g} / \mathrm{m}^{2}$. Above $500 \mathrm{~Hz}$ the improvement is in the order of 0.2 . The corresponding optimised values are stated in Table 2 and 3. 


\section{Conclusion}

There are different approaches to analyze the behavior of multilayer absorbers. It has been shown that the equivalent circuit method (EC) with correct modeling of the air cavities by distributed elements and the impedance transfer method (ITM) yield identical impedance relations. However, the equivalent circuit method (EC) has the advantage of offering additional information about sound field quantities at specific points inside the structure. For example, transfer functions can be determined to evaluate sound transmission losses or absorption coefficients can be calculated from considerations of power dissipation. Although a couple of recent papers question its accuracy, the EC method is a reliable and universal tool, provided that the distributed elements are properly applied.

In the proposed theoretical model for lightweight curtains, the fabric sheets are represented by a network of discrete impedances describing soundinduced vibrations and the airflow through the fabric. For limp materials, typically only inertia is taken into account which reduces the mechanical impedance of each fabric to an inductor in the equivalent electrical circuit. The total airflow through each fabric is assumed to consist of the interyarn and the intrayarn airflow-both represented by specific airflow resistances. For the interyarn airflow, a capillary model with geometrical parameters is adopted.

For curtains with an acoustically hard backing, formulas for the oblique incidence absorption coefficient and the statistical absorption coefficient measured in the reverberation chamber have been derived. The formula for the statistical absorption coefficient considers the edge effect and accounts for the non-uniform sound intensity distribution under measurement conditions. For curtains that are not placed in the vicinity of a room surface, i.e. freely hanging curtains, the oblique incident absorption coefficient is given. However, for the statistical case, the equivalent sound absorption area is used as a concept to compare different curtain layouts. For the single sheet curtain, simplified formulas were deduced.

The model was validated by measurements on a set of 24 lightweight, woven fabrics consisting of different synthetic yarns. Geometrical parameters such as the interyarn porosity and the hydraulic pore radius were extracted from macro photographs. The employed fabrics cover a large range of interyarn porosities, pore aspect ratios and specific airflow resistances. Each fabric was measured in an impedance tube with two different air cavities. Measured 
and - based on geometrical parameters - predicted normal incidence absorption coefficients exhibit excellent agreement, with mean value and standard deviation of the differences of $0.01 \pm 0.05$. Compared to predictions by a former, simpler model [6], the standard deviation of the errors could be reduced by a factor of 2 by considering the intrayarn airflow and the usage of a more refined model for the interyarn airflow. From this agreement it can be concluded that the considered absorption coefficients of this set of fabrics are elicited predominantly by viscous and frame motion effects - and that acoustical inertia and thermal effects are of minor importance.

The proposed prediction model was used to investigate several questions arising from practical applications of lightweight multilayer curtains as sound absorbers. The following conclusions can be drawn: (1) Sound-induced vibrations of the fabrics are an acoustically relevant aspect in the design of lightweight curtains. (2) The optimal specific airflow resistances of the individual layers may not be given in a compact analytical form but have to be determined by an optimisation procedure. (3) The sound energy absorbed by curtains placed in the diffuse field of a room is in the same order of magnitude as the absorption by comparable curtains mounted in front of a wall. (4) For given mass and extension, multilayer arrangements perform superiorly to single layer curtains.

Further research has to be carried out into the modeling of draped curtains and the mechanical behavior of fabrics under clamped boundary conditions.

\section{Appendix A. Transfer matrix method (TMM)}

A powerful method to calculate multilayer structures is the transfer matrix method. An excellent overview can be found in the book by Allard and Atalla [18]. Sound pressure, $p$, and the x-component of the particle velocity, $v$, are arranged in a row vector $\mathbf{V}=\left[\begin{array}{ll}p & v\end{array}\right]^{T}$ where $T$ denotes transposition. The acoustic field on the left-hand end of a medium, $\mathbf{V}_{l}$, is calculated by $\mathbf{V}_{l}=\mathbf{T} \cdot \mathbf{V}_{r}$ where $\mathbf{V}_{r}$ is the acoustic field on the right-hand end of this medium and $\mathbf{T}$ denotes a $2 \times 2$ transfer matrix.

According to [18] air layers are represented by a fluid layer transfer matrix given by

$$
\mathbf{T}_{a i r, i}=\left[\begin{array}{cc}
\cos \left(k_{0} \cos \theta d_{i}\right) & j \frac{Z_{0}}{\cos \theta} \sin \left(k_{0} \cos \theta d_{i}\right) \\
j \frac{\cos \theta}{Z_{0}} \sin \left(k_{0} \cos \theta d_{i}\right) & \cos \left(k_{0} \cos \theta d_{i}\right)
\end{array}\right]
$$


The transfer matix of the fabrics is given as

$$
\mathbf{T}_{\text {fabric }, i}=\left[\begin{array}{cc}
1 & Z_{f, i} \\
0 & 1
\end{array}\right]
$$

In order to evaluate the surface impedance the backing can be represented by the row vector

$$
\mathbf{B}=\left[\begin{array}{c}
1 \\
1 / Z_{t}
\end{array}\right]
$$

The field at the input of the curtain is then given by

$$
\mathbf{V}^{\prime}=\mathbf{T}_{f a b r i c, 1} \cdot \mathbf{T}_{a i r, 1} \cdots \mathbf{T}_{\text {fabric }, N} \cdot \mathbf{T}_{a i r, N} \cdot \mathbf{B}
$$

By division of the first and the second element of Eq. (A.4) one obtains the surface impedance

$$
Z_{s}=\frac{\mathbf{V}^{\prime}(1)}{\mathbf{V}^{\prime}(2)}
$$

of the curtain system. This method delivers the identical expression for $Z_{s}$ as the EC method and the ITM. If the curtain is terminated by air also the transmission coefficient can be evaluated using the TMM. By arbitrarily setting $\check{p}_{0}=1$ and assuming an infinitely extended system, the acoustic field at the input of the structure is given by

$$
\mathbf{V}_{1}=\left[\begin{array}{c}
Z_{s} \\
1
\end{array}\right] \cdot \frac{2}{Z_{s}+Z_{0} / \cos \theta}
$$

The field at the input of air layer $N$ thus amounts to

$$
\mathbf{V}_{N}=\mathbf{T}_{\text {fabric }, N}^{-1} \cdots \mathbf{T}_{\text {air }, 1}^{-1} \cdot \mathbf{T}_{\text {fabric }, 1}^{-1} \cdot \mathbf{V}_{1}
$$

and the transmission coefficient is given by the first element of Eq. (A.7)

$$
\tau=\mathbf{V}_{N}(1) .
$$




\section{Appendix B. Field impedance of a rectangular absorber}

The field impedance $Z_{F}$ (or radiation impedance) depends on the size and shape of the absorber as well as the angle of incidence $\theta$, and the azimuth angle $\varphi$. If $Z_{F}$ only weakly depends on $\varphi$, i.e. the shape of the absorber is approximately circular, an averaged field impedance $\bar{Z}_{F}$

$$
\bar{Z}_{F}=\frac{1}{2 \pi} \int_{0}^{2 \pi} Z_{F} d \varphi
$$

can be used in order to predict the statistical absorption coefficient. For an infinitely extended absorber $Z_{F}=1 / \cos \theta$. However, for a finite absorber $Z_{F}$ differs from $1 / \cos \theta$, in particular near grazing incidence. The field impedance for a finite absorber is given by [40]

$$
Z_{F}=\frac{j k_{0}}{S} \int_{S} \int_{S} G e^{j k_{0}\left[\mu_{x}\left(x^{\prime}-x\right)+\mu_{y}\left(y^{\prime}-y\right)\right]} d x d y d x^{\prime} d y^{\prime}
$$

with the surface area of the absorber $S, \mu_{x}=\sin \theta \cos \varphi, \mu_{y}=\sin \theta \sin \varphi$, Green's function $G=(2 \pi R)^{-1} e^{j k_{0} R}$ and $R=\sqrt{\left(x-x^{\prime}\right)^{2}+\left(y-y^{\prime}\right)^{2}}$. For a rectangular shape - as it is common for measurements of the statistical absorption coefficient in the reverberation chamber-Eq. (B.2) can be analytically simplified into an expression with only one integral. In [45] the formula for the real part of $Z_{F}$ is derived. As for the calculation of the absorption coefficient also the imaginary part of $Z_{F}$ is required, the formulas from [45] have been extended and are given here in a generalized form for complex-valued $Z_{F}$.

Let $L_{x}$ and $L_{y}$ denote the length and width of the absorber with aspect ratio $r=L_{x} / L_{y}$. By using several variable substitutions (see [45]) Eq. (B.2) can be simplified into

$$
Z_{F}=\frac{k_{0} L_{x}}{16 \pi} \int_{0}^{\pi / 2} \sum_{i=1}^{4} F\left(\psi, \alpha_{i}\right) d \psi
$$

with auxiliary variables 


$$
\begin{aligned}
F\left(\psi, \alpha_{i}\right) & =\frac{4}{\alpha_{i}}-\frac{2 a}{\alpha_{i}^{3}}+j \frac{2 b}{\alpha_{i}^{2}}+e^{-j R_{\theta} \alpha_{i}} \cdot C \\
C & =-\frac{4}{\alpha_{i}}+2 b\left(\frac{R_{\theta}}{\alpha_{i}}-j \frac{1}{\alpha_{i}^{2}}\right)+a\left(\frac{2}{\alpha_{i}^{3}}+j \frac{2 R_{\theta}}{\alpha_{i}^{2}}-\frac{R_{\theta}^{2}}{\alpha_{i}}\right) \\
a & =r \cos \psi \sin \psi \\
b & =\cos \psi+r \sin \psi \\
\alpha_{1,2} & =\frac{k_{0} L_{x}}{2}[1 \pm \sin \theta \cos (\psi-\varphi)] \\
\alpha_{3,4} & =\frac{k_{0} L_{x}}{2}[1 \pm \sin \theta \cos (\psi+\varphi)]
\end{aligned}
$$

and

$$
R_{\theta}= \begin{cases}\frac{2}{\cos \theta} & \text { for } \theta \leq \arctan \frac{1}{r} \\ \frac{2}{r \sin \theta} & \text { else }\end{cases}
$$

The integral in Eq. (B.3) can be easily solved numerically by substituting $\kappa=(4 \psi / \pi)-1$ and subsequently applying a Gauss numerical integration scheme.

\section{Acknowledgment}

This work was supported by the Swiss Innovation Promotion Agency (CTI) [grant number 10675.1 PFIW-IW].

[1] V. M. A. Peutz, Sound absorption of curtains, in: 79th Meeting of the Acoustical Society of America, Atlantic City, 1970.

[2] Y. Shoshani, G. Rosenhouse, Noise absorption by woven fabrics, Applied Acoustics 30 (4) (1990) 321-333.

[3] J. Kang, H. V. Fuchs, Predicting the absorption of open weave textiles and micro-perforated membranes backed by an air space, Journal of Sound and Vibration 220 (5) (1999) 905-920.

[4] T. Dias, R. Monaragala, Sound absorbtion in knitted structures for interior noise reduction in automobiles, Measurement Science \& Technology 17 (9) (2006) 2499-2505. 
[5] F. Chevillotte, Controlling sound absorption by an upstream resistive layer, Applied Acoustics 73 (1) (2012) 56-60.

[6] R. Pieren, Sound absorption modeling of thin woven fabrics backed by an air cavity, Textile Research Journal 82 (9) (2012) 864-874.

[7] K. Horoshenkov, K. Sakagami, M. Morimoto, On the dissipation of acoustic energy in a thin, infinite, poroelastic plate, Acta Acustica united with Acustica 88 (4) (2002) 500-506.

[8] K. Horoshenkov, K. Sakagami, A method to calculate the acoustic response of a thin, baffled,simply supported poroelastic plate, Journal of the Acoustical Society of America 110 (2) (2001) 904-917.

[9] K. Sakagami, M. Kiyama, M. Morimoto, D. Takahashi, Detailed analysis of the acoustic properties of a permeable membrane, Applied Acoustics 54 (2) (1998) 93-111.

[10] D. Y. Maa, Microperforated-panel wideband absorbers, Noise Control Engineering Journal 29 (3) (1987) 77-84.

[11] D. Y. Maa, Potential of microperforated panel absorber, Journal of the Acoustical Society of America 104 (5) (1998) 2861-2866.

[12] K. Sakagami, T. Nakamori, M. Morimoto, M. Yairi, Double-leaf microperforated panel space absorbers: A revised theory and detailed analysis, Applied Acoustics 70 (5) (2009) 703-709.

[13] J. Zou, Y. Shen, J. Yang, X. Qiu, A note on the prediction method of reverberation absorption coefficient of double layer micro-perforated membrane, Applied Acoustics 67 (2) (2006) 106-111.

[14] K. Sakagami, M. Morimoto, W. Koike, A numerical study of doubleleaf microperforated panel absorbers, Applied Acoustics 67 (7) (2006) 609-619.

[15] H. Ruiz, P. Cobo, F. Jacobsen, Optimization of multiple-layer microperforated panels by simulated annealing, Applied Acoustics 72 (10) (2011) $772-776$. 
[16] K. Sakagami, Y. Fukutani, M. Yairi, M. Morimoto, A theoretical study on the effect of a permeable membrane in the air cavity of a doubleleaf microperforated panel space sound absorber, Applied Acoustics 79 (2014) 104-109.

[17] D. Brouard, B. Lafarge, A. J.-F., A general method of modelling sound propagation in layered media, Journal of Sound and Vibration 183 (1) (1995) 129-142.

[18] J. F. Allard, N. Atalla, Propagation of Sound in Porous Media: Modelling Sound Absorbing Materials, Wiley, Chichester, 2009.

[19] L. Brekhovskikh, Waves in Layered Media, 2nd Edition, Applied Mathematics and Mechanics, Vol. 16, Academic Press, New York, 1980.

[20] P. Cobo, H. Ruiz, J. Alvarez, Double-layer microperforated panel/porous absorber as liner for anechoic closing of the test section in wind tunnels, Acta Acustica United with Acustica 96 (5) (2010) 914922.

[21] P. Cobo, M. Cuesta, M. Siguero, Comparison of models describing double layer microperforated absorbers, Noise Control Engineering Journal 57 (1) (2009) 10-15.

[22] J. Liu, W. Bao, L. Shi, B. Zuo, W. Gao, General regression neural network for prediction of sound absorption coefficients of sandwich structure nonwoven absorbers, Applied Acoustics 76 (2014) 128-137.

[23] K. Sakagami, K. Matsutani, M. Morimoto, Sound absorption of a double-leaf micro-perforated panel with an air-back cavity and a rigidback wall: Detailed analysis with a helmholtz-kirchhoff integral formulation, Applied Acoustics 71 (5) (2010) 411-417.

[24] R. Pieren, Sound absorption modelling of thin, lightweight curtains, in: Proceedings Euronoise Prague, Prague, 2012, pp. 815-820.

[25] R. Del Rey, J. Alba, M. Blanes, B. Marco, The acoustic absorption of textile curtains on the function of the fullness, MATERIALES DE CONSTRUCCION 63 (312) (2013) 569-580.

[26] F. P. Mechel, Formulas of Acoustics, Vol. 2nd ed., Springer, Berlin, 2008. 
[27] J. Lee, G. Swenson, Compact sound absorbers for low frequencies, Noise Control Engineering Journal 38 (1992) 109-117.

[28] Y. Lee, E. Lee, Widening the sound absorption bandwidths of flexible micro-perforated curved absorbers using structural and acoustic resonances, Int. Journal of Mechanical Sciences 49 (2007) 925-934.

[29] J. Carbajo, W. Cardenas, J. Torres, J. Ramis, R. Del Rey, An experimental study of different profile micro-perforated panels (mpps) used in audio communication systems, in: Proceedings Euronoise Prague, Prague, 2012, pp. 854-858.

[30] N. Atalla, F. Sgard, Modeling of perforated plates and screens using rigid frame porous models, Journal of Sound and Vibration 303 (1-2) (2007) 195-208.

[31] I. O. of Standardization, Iso 9053: Acoustics - materials for acoustical applications - determination of airflow resistance (1991).

[32] L. Jaouen, F. X. Becot, Acoustical characterization of perforated facings, Journal of the Acoustical Society of America 129 (3) (2011) 1400-1406.

[33] Y. P. Liu, H. Hu, Sound absorption behavior of knitted spacer fabrics, Textile Research Journal 80 (18) (2010) 1949-1957.

[34] M. G. Honarvar, A. A. A. Jeddi, M. A. Tehran, Noise absorption modeling of rib knitted fabrics, Textile Research Journal 80 (14) (2010) 13921404 .

[35] R. Christensen, Modeling the effects of viscosity and thermal conduction on acoustic propagation in rigid tubes with various cross-sectional shapes, Acta Acustica United with Acustica 97 (2) (2011) 193201.

[36] M. Moeser, W. Kropp, Koerperschall - Physikalische Grundlagen und technische Anwendungen, Springer, Berlin Heidelberg, 2010.

[37] R. L. Mu, M. Toyoda, D. Takahashi, Sound insulation characteristics of multi-layer structures with a microperforated panel, Applied Acoustics 72 (11) (2011) 849-855. 
[38] M. Toyoda, K. Sakagami, D. Takahashi, M. Morimoto, Effect of a honeycomb on the sound absorption characteristics of panel-type absorbers, Applied Acoustics 72 (2011) 943-948.

[39] F. P. Mechel, Schallabsorber - Band III: Anwendungen, S. Hirzel Verlag, Stuttgart, 1998.

[40] S.-I. Thomasson, On the absorption coefficient, Acustica 44 (1980) 265273.

[41] H. Kuttruff, Room Acoustics, Applied Science Publishers Ltd, 1973, 0-85334-573-2.

[42] T. J. Cox, P. D'Antonio, Acoustic Absorbers and Diffusers - Theory, Design and Application, Spon Press, 2004, 0-415-29649-8.

[43] C.-H. Jeong, Non-uniform sound intensity distributions when measuring absorption coefficients in reverberation chambers using a phased beam tracing, The Journal of the Acoustical Society of America 127 (6) (2010) $3560-3568$.

[44] I. O. of Standardization, Iso 354: Acoustics - measurement of sound absorption in a reverberation room (2003).

[45] D. Rhazi, N. Atalla, A simple method to account for size effects in the transfer matrix method, The Journal of the Acoustical Society of America 127 (2) (2010) EL30-EL36.

[46] C. H. Jeong, Guideline for adopting the local reaction assumption for porous absorbers in terms of random incidence absorption coefficients, Acta Acustica United with Acustica 97 (5) (2011) 779-790.

[47] I. O. of Standardization, Iso 10534-2: Acoustics - determination of sound absorption coefficient and impedance in impedance tubes, part 2: Transfer-function method (1998).

[48] I. O. of Standardization, Iso/iec guide 98-3: Uncertainty of measurement - part 3: Guide to the expression of uncertainty in measurement (gum:1995) (2008).

[49] B. Schäffer, S. Plüss, G. Thomann, Estimating the model-specific uncertainty of aircraft noise calculations, Applied Acoustics 84 (2014) 58-72. 